ARTICLE

Received 11 May 2015 | Accepted 7 Aug 2015 | Published 15 Sep 2015

DOI: $10.1038 /$ ncomms 9314

OPEN

\title{
How Leiomodin and Tropomodulin use a common fold for different actin assembly functions
}

Malgorzata Boczkowska ${ }^{1, \star}$, Grzegorz Rebowski ${ }^{1, \star}$, Elena Kremneva ${ }^{2, \star}$, Pekka Lappalainen $^{2}$ \& Roberto Dominguez ${ }^{1}$

How proteins sharing a common fold have evolved different functions is a fundamental question in biology. Tropomodulins (Tmods) are prototypical actin filament pointed-endcapping proteins, whereas their homologues, Leiomodins (Lmods), are powerful filament nucleators. We show that Tmods and Lmods do not compete biochemically, and display similar but distinct localization in sarcomeres. Changes along the polypeptide chains of Tmods and Lmods exquisitely adapt their functions for capping versus nucleation. Tmods have alternating tropomyosin (TM)- and actin-binding sites (TMBS1, ABS1, TMBS2 and ABS2). Lmods additionally contain a C-terminal extension featuring an actin-binding WH2 domain. Unexpectedly, the different activities of Tmods and Lmods do not arise from the Lmodspecific extension. Instead, nucleation by Lmods depends on two major adaptations-the loss of pointed-end-capping elements present in Tmods and the specialization of the highly conserved ABS2 for recruitment of two or more actin subunits. The $\mathrm{WH} 2$ domain plays only an auxiliary role in nucleation.

\footnotetext{
${ }^{1}$ Department of Physiology, Perelman School of Medicine, University of Pennsylvania, Philadelphia, Pennsylvania 19104, USA. ${ }^{2}$ Institute of Biotechnology, PO Box 56, 00014 University of Helsinki, Helsinki, Finland. * These authors contributed equally to this work. Correspondence and requests for materials should be addressed to R.D. (email: droberto@mail.med.upenn.edu).
} 
T ropomodulins (Tmods) constitute a conserved family of four isoforms that work in conjunction with one of several tropomyosin (TM) isoforms to cap the pointed end of actin filaments in cytoskeletal structures characterized by their uniform distribution of the lengths of actin filaments ${ }^{1}$. These structures include the sarcomere of cardiac and skeletal muscle cells and the spectrin-based membrane skeleton ${ }^{1,2}$. The unique domain organization of Tmods is precisely matched to their core function in pointed end capping, and consists of alternating TMand actin-binding sites (TMBS1, ABS1, TMBS2 and ABS2). While TMBS1, ABS1 and TMBS2 feature helical segments, they lie within the otherwise unstructured $\mathrm{N}$-terminal $\sim 160$-aminoacid (aa) region of $\mathrm{Tmod}^{3-5}$. In contrast, most of ABS2, comprising the C-terminal $\sim 200$-aa region of Tmods, is folded as a leucine-rich repeat (LRR) domain ${ }^{6}$. We recently showed that ABS1 binds on top of the first actin subunit at the pointed end of the actin filament, adopting an extended but ordered structure, whereas ABS2 binds at the interface between the first three subunits of the filament, interacting mostly with the second subunit?

Leiomodins (Lmods) are related to Tmods, and constitute a subfamily of three isoforms: Lmod1, expressed preferentially in differentiated smooth muscle cells; and Lmod2 and Lmod3, expressed more abundantly in skeletal and cardiac muscles ${ }^{8-12}$. Lmods are distinguished from Tmods by the presence of a C-terminal extension, featuring a proline-rich region and a $\mathrm{WH} 2$ domain. The $\mathrm{WH} 2$ domain is a widespread actin monomerbinding motif ${ }^{13,14}$, frequently found C-terminally to proline-rich regions among cytoskeletal proteins that nucleate actin polymerization ${ }^{15}$. The unique characteristics of the C-terminal extension suggested that Lmods could function as actin filament nucleators, which we initially demonstrated for Lmod2 (ref. 11), and was recently also shown for Lmod3 (ref. 10). Emphasizing the physiological importance of Lmods, recent studies have established that Lmod3 deficiency results in nemaline myopathy in mice ${ }^{16}$, and human patients affected by an unusually lethal form of this disease carry mutations in the gene encoding Lmod3 (ref. 10).

The main feature defining an actin filament nucleator is the presence of multiple actin-binding sites, allowing these molecules to recruit two or more actin subunits to form a polymerization nucleus (or seed). Therefore, we had initially proposed that the C-terminal extension, absent in Tmods, was the main factor responsible for the nucleation activity of Lmods, since it could mediate the recruitment of an additional actin subunit through the WH2 domain ${ }^{11}$. However, our recent finding that the ABS2 of Tmods contacts three actin subunits in the filament ${ }^{7}$ raised two important questions: (a) why are Tmods unable to nucleate polymerization if they can interact with up to three actin subunits at the pointed end of the filament? and (b) does ABS2 play a more prominent role in Lmod-based nucleation than previously anticipated? By addressing these questions here, we made several important findings. We show that $\mathrm{ABS2}$, and not the C-terminal extension, is the main factor distinguishing Lmods and Tmods as filament nucleators and pointed-end-capping proteins, respectively. This was a surprising finding since ABS2 is also the most highly conserved region among these proteins. We further show that, through a series of local changes along the polypeptide chain, Lmods have lost specific features that allow Tmods to cap the pointed end, while acquiring new features required for nucleation. Consistently, we established that Tmods and Lmods do not compete biochemically with each other, and display similar but distinct localization in muscle sarcomeres. Finally, we found that the WH2 domaincontaining extension of Lmods plays only an auxiliary role in nucleation.

\section{Results}

Smooth muscle Lmod1 is a filament nucleator. Lmod isoforms differ considerably, and Lmod1 is the most divergent of the three isoforms (Fig. 1a and Supplementary Fig. 1a). The region $\mathrm{N}$-terminal to ABS2 of Lmod1 comprises $\sim 300$ aa, which is much longer than in Tmods or other Lmods, whereas its C-terminal extension is shorter than in other Lmods. While the striated muscle isoforms Lmod2 (ref. 11) and Lmod3 (ref. 10) have been shown to nucleate actin polymerization in vitro, smooth muscle Lmod1 remains uncharacterized, and problems with protein degradation have hampered its study. Here we obtained pure proteins by using $\mathrm{N}$ - and C-terminal affinity tags followed by gel filtration (Methods and Supplementary Fig. 2), and proteins were used in experiments within 3 days after purification. Using the pyrene actin polymerization assay, we found that Lmod1 had strong nucleation activity, comparable to that of Arp2/3 complex and Lmod2, and this activity increased with Lmod1 concentration (Fig. 1b). We thus conclude that despite extensive differences between Lmod isoforms, they all share a common ability to strongly activate actin polymerization in vitro, and thus the elements necessary for this activity must be conserved among isoforms.

\section{Lmod and Tmod domains in filament nucleation versus capping.} Tmod1 has strong pointed-end-capping activity 7,17 but, as confirmed here (Fig. 1b), it completely lacks nucleation activity over a range of concentrations. For this reason, we had previously proposed that the C-terminal extension of Lmods, which is missing in Tmods, was the main factor responsible for their nucleation activity ${ }^{11}$. To more directly test this hypothesis, we asked whether adding the C-terminal extension of human Lmod2 C terminally to human Tmod1 would be sufficient to produce a filament nucleator. Note also that the sequence of human Lmod2 was corrected in databases since the submission of our original work $^{11}$, and the corrected sequence used here includes changes both N and C terminal to ABS2 (see Methods and Supplementary Fig. 1). To our surprise, however, the hybrid construct Tmod1Lmod2 $2_{C}$ (Fig. 1a), displayed limited nucleation activity, which increased only slightly with concentration, and was significantly lower than that of either Lmod1 or Lmod2 (Fig. 1b). Conversely, removing the C-terminal extension of Lmod2 lowered its nucleation activity, but the resulting construct $\left(\operatorname{Lmod} 2_{\mathrm{N}-\mathrm{ABS} 2}\right)$ was a more powerful nucleator over a rage of concentrations than Tmod1 with addition of the C-terminal extension (Fig. 1c).

These results suggest that regions other than the C-terminal extension are the main source of the functional differences between Lmods and Tmods. To more precisely map the source of these differences, we deleted the region $\mathrm{N}$-terminal to ABS2 of Lmod2 (construct Lmod2 $2_{\mathrm{ABS2}-\mathrm{C}}$ ), which resulted in a relatively minor drop in activity (Fig. 1c). What is more, the isolated ABS2 of Lmod2 still retained substantial nucleation activity, which increased with concentration (Fig. 1c). Similar results were obtained with Lmod1, although in this case removing the $\mathrm{N}$-terminal region had nearly no effect on the nucleation activity, and the isolated ABS2 had even stronger activity than that of Lmod2 (Fig. 1d).

Other than the $\mathrm{WH} 2$ domain, the C-terminal extension of Lmods contains a proline-rich region, which is a potential profilin-actin-binding site. Thus, we tested the effect of profilin on Lmod1-mediated nucleation (Supplementary Fig. 3). Profilin is known to inhibit spontaneous actin nucleation and pointedend elongation ${ }^{18}$. Similarly, profilin inhibited in a concentrationdependent manner the nucleation activity of both $\operatorname{Lmod} 1_{\mathrm{FL}}$ and $\operatorname{Lmod} 1_{\mathrm{ABS} 2}$, lacking the proline-rich region. We thus conclude that, as previously observed with Lmod2 (ref. 11), the proline-rich 


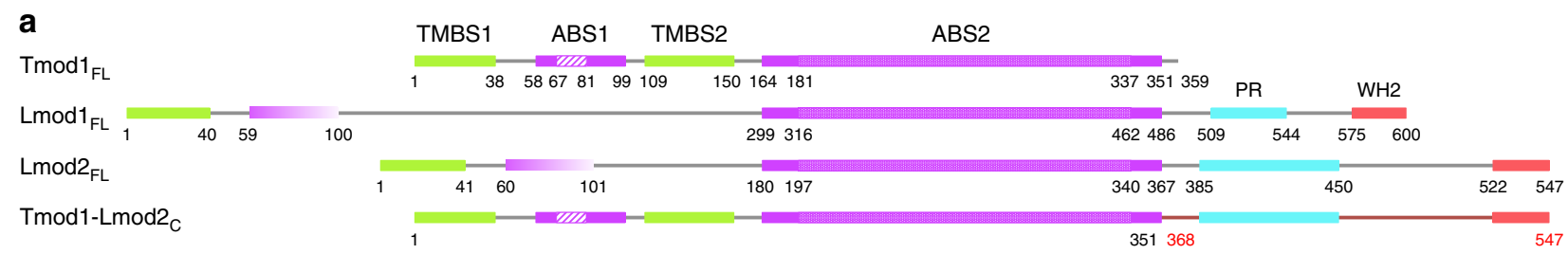

b

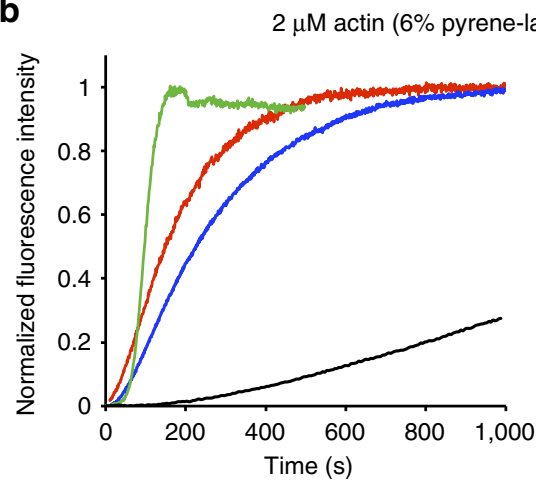

Buffer

C

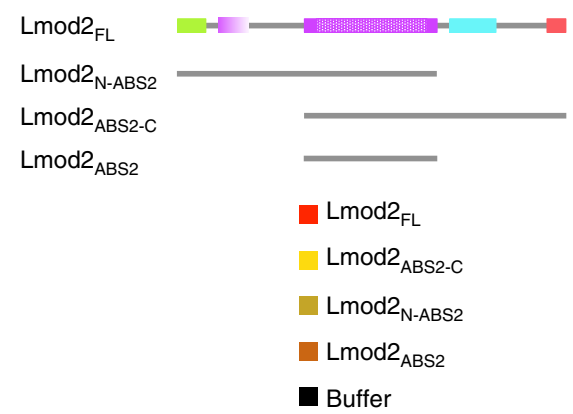

d

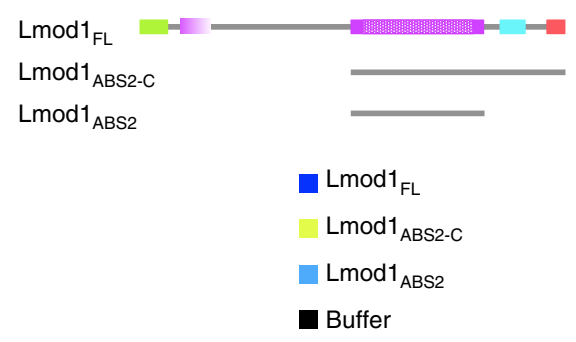

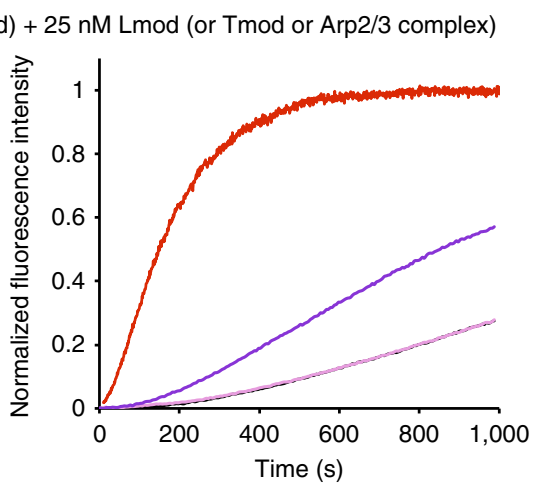

Lmod1 $1_{\mathrm{FL}}$

Lmod2 $2 \mu \mathrm{M}$ actin $(6 \%$ pyrene-labeled $)+25 \mathrm{nM} \operatorname{Lmod} 2$

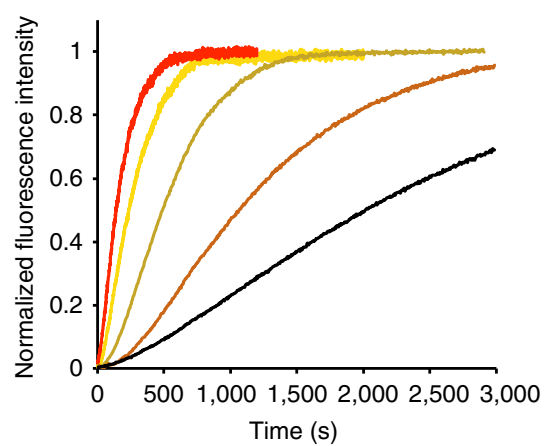

$2 \mu \mathrm{M}$ actin (6\% pyrene-labeled) $+25 \mathrm{nM}$ Lmod1

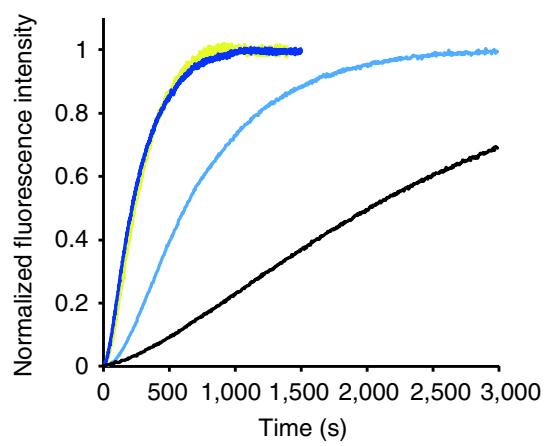

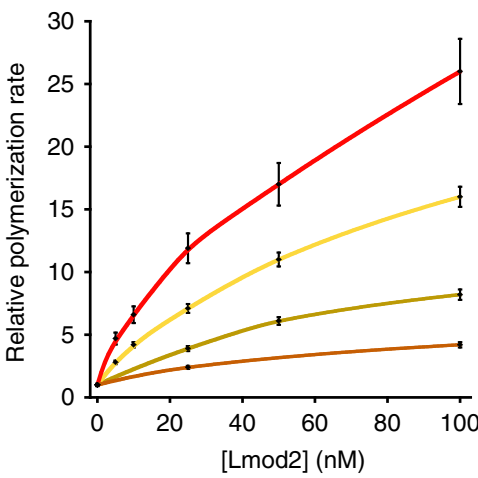

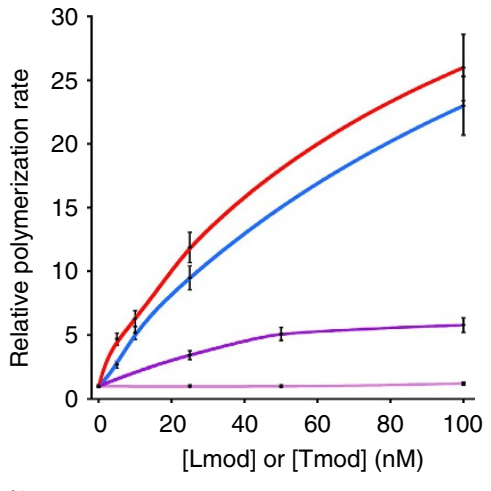

Tmod1-Lmod2

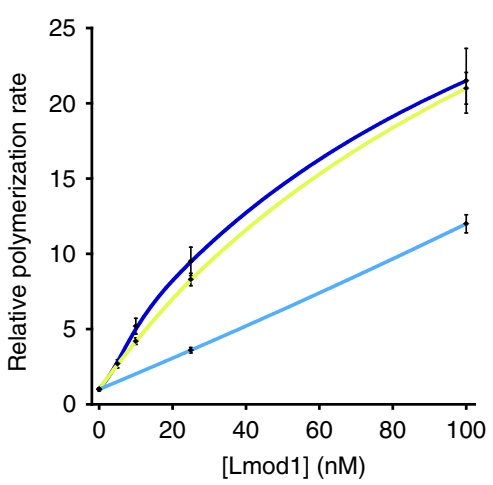

Figure 1 Domains of Lmod and Tmod and actin nucleation. (a) Domain organization of Tmod1 and Lmods, and design of the Tmod1-Lmod2 $2_{c}$ hybrid construct. Numbers under the diagrams indicate the boundaries of domains. For Tmod1, the helix of ABS1 (aa 67-81) and the LRR portion of ABS2 (aa 181-337) are highlighted. (b) Nucleation activity of full-length Lmod1, Lmod2 and the hybrid construct Tmod1-Lmod2 as compared with Tmod1 and the Arp2/3 complex ( $25 \mathrm{nM}$, activated by $100 \mathrm{nM} \mathrm{N-WASP} \mathrm{WCA).} \mathrm{The} \mathrm{left} \mathrm{two} \mathrm{graphs} \mathrm{show} \mathrm{time} \mathrm{courses} \mathrm{of} \mathrm{polymerization} \mathrm{of} 2 \mu \mathrm{M}$ Mg-ATP-actin ( $6 \%$ pyrene labelled) in the presence of $25 \mathrm{nM}$ of the indicated proteins (colour coded) or the buffer control (black). The graph on the right shows the concentration dependence of the polymerization rates, displayed as the mean of three experiments \pm s.e.m. (c,d) Contribution of the various domains of Lmod1 (c) and Lmod2 (d) to the nucleation activity. The graphs on the left and the right show, respectively, the time course of polymerization of $2 \mu \mathrm{M}$ $\mathrm{Mg}$-ATP-actin in the presence of $25 \mathrm{nM}$ Lmod fragments (colour coded) or buffer (black) and the concentration dependence of polymerization rates.

region of Lmod1 is not implicated in the recruitment of profilin-actin for nucleation.

Together, these results suggest that the C-terminal extension plays a secondary role in Lmod-mediated nucleation, whereas
ABS2 emerges as the main source of the differences between Lmods and Tmods. This was a surprising finding, because ABS2 is also the most highly conserved region among these proteins (Supplementary Fig. 1). 
Role of the $\mathrm{N}$-terminal region in nucleation versus capping. Tmods bind TM through two different sites ${ }^{5}$, and this interaction enhances their pointed-end-capping efficiency ${ }^{4,7,17}$. Comparison of 50 Tmod and 50 Lmod sequences separately and together shows that only the first of these sites in conserved in Lmods (Fig. 2a and Supplementary Figs 1 and 4). While we showed above that in the absence of TM N-terminal deletions had a relatively minor effect on the nucleation activity of Lmods, we previously found that TM increases the activity of $\operatorname{Lmod} 2_{\mathrm{FL}}$ (refs 11,19). A similar effect was observed here over a range of concentrations with $\operatorname{Lmod}_{\mathrm{FL}}$ (Fig. 2b).

It was previously thought that ABS1 in Tmods consisted mostly of an $\alpha$-helix ${ }^{1}$, which appeared to be conserved in Lmods ${ }^{11}$. However, we recently found that ABS1 is longer than anticipated, comprising in addition to the $\alpha$-helix an extended region that binds along subdomains 2 and 1 of the first actin subunit of the filament ${ }^{7}$. Sequence analysis reveals that only the $\mathrm{N}$-terminal portion of ABS1 is somewhat conserved in Lmods (Fig. 2a and Supplementary Figs 1 and 4), which together with the minor effect of N-terminal deletions described above (Fig. 1c,d) made us question whether this interaction was conserved in Lmods. Consistent with our previous findings ${ }^{7}, \mathrm{Tmod}_{\mathrm{ABS} 1}$ (Fig. 2a) bound one actin monomer (stabilized with LatB) with micromolar affinity by isothermal titration calorimetry (ITC) (Fig. 2c). In contrast, the equivalent constructs of Lmod1 and Lmod2 did not bind LatB-actin. We thus conclude that the interaction of ABS1 with actin, which is critical for pointed-end filament capping by Tmods ${ }^{4,7}$, is not conserved in Lmods and may not be required for nucleation.

Fine-tuning ABS2 for nucleation versus capping. While ABS2 is generally well conserved among Tmods and Lmods, we noticed
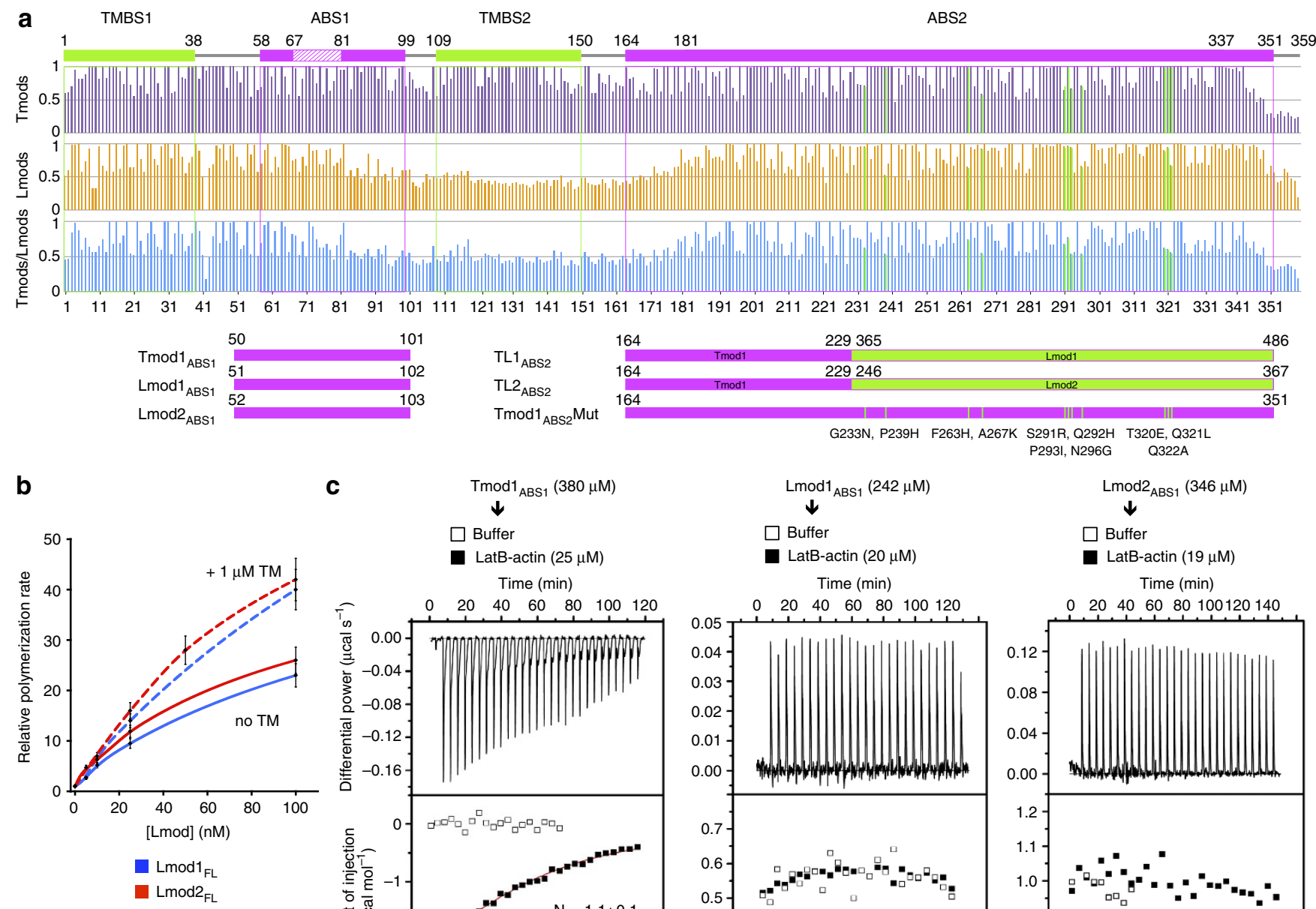

C
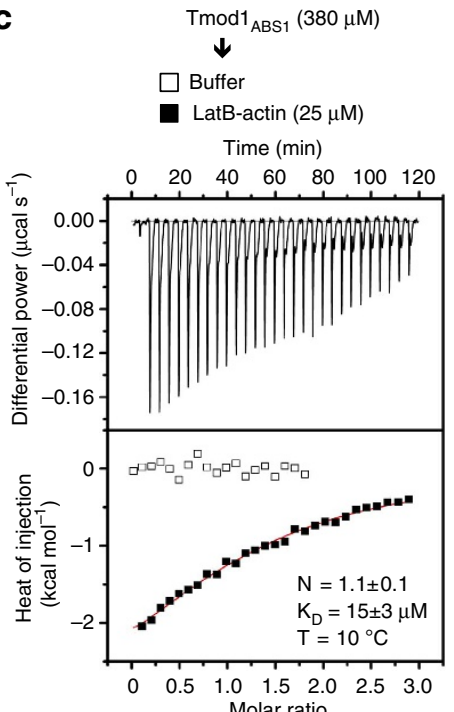

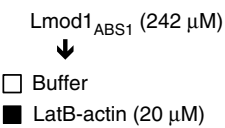

Time ( $\mathrm{min})$

$\begin{array}{lllllll}0 & 20 & 40 & 60 & 80 & 100 & 120\end{array}$

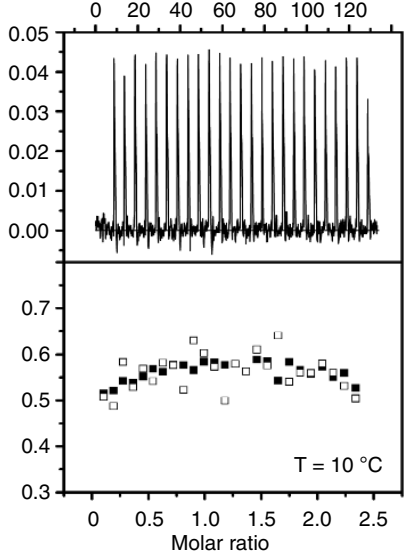

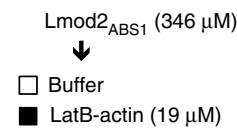

Time (min)

$0 \quad 204060 \quad 80100120140$

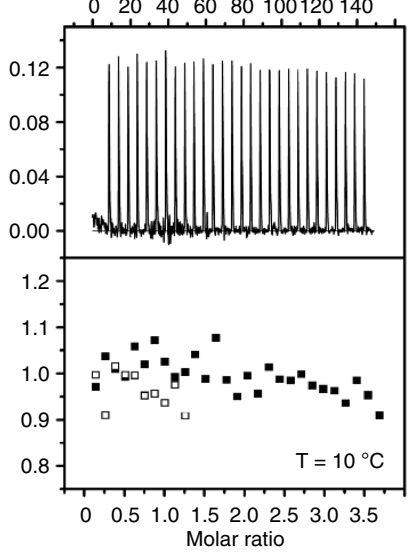

Figure 2 | The different activities of Tmod and Lmod result from differences in their interactions with actin and TM. (a) Sequence conservation analysis of Tmod and Lmod (see also Supplementary Fig. 1). Fifty Tmod (purple) and fifty Lmod (orange) sequences were aligned separately or together (blue), and residue conservation scores were calculated with the program Scorecons ${ }^{29}$ and plotted on the human Tmod1 sequence (the scores of residues absent in Tmod1 are not shown). The Tmod1 diagram on top indicates the boundaries of the TM- and actin-binding sites. Diagrams on the bottom illustrate ABS1 constructs, and hybrid Tmod1 (magenta)/Lmod (green) ABS2 constructs ( $T L 1_{A B S 2}, T_{2} 2_{A B S 2}$ and $\operatorname{Tmod1}_{\mathrm{ABS} 2} \mathrm{Mut}_{\text {). The } 11 \text { residues of Tmod1 }} \mathrm{ABS}_{2}$ replaced by their Lmod1 counterparts (highlighted in green across the conservation plots) tend to be conserved among Lmods, but poorly conserved between Lmods and Tmods. (b) Concentration dependence of polymerization rates of Lmod1 and Lmod2 in the absence (solid lines) or the presence (broken lines) of $1 \mu \mathrm{M} \mathrm{TM}$, displayed as the mean of three experiments \pm s.e.m. (c) ITC titrations of ABS1 constructs (as indicated) into LatB-actin. The experimental conditions are listed for each experiment, including temperature and the concentrations of ABS1 constructs in the syringe and LatB-actin in the cell. Open symbols correspond to titrations into buffer. Only the titration of $\operatorname{Tmod1}_{\mathrm{ABS} 1}$ could be fitted to a binding isotherm (red curve, fitting parameters inside graph), whereas $L \operatorname{Lmod1} 1_{\mathrm{ABS} 1}$ and $\operatorname{Lmod} 2_{\mathrm{ABS} 1}$ did not appear to bind (solid black symbols). Errors correspond to the s.d. of the fits. 
that sequence conservation drops sharply at the $\mathrm{N}$ - and C-terminal ends of the domain (Fig. 2a and Supplementary Figs 1 and 4). The differences at the C-terminal end appeared less critical for function, because Tmod 1 residues that interact with actin (up to Arg-343) (ref. 7) tend to be conserved in Lmods. However, the variable region at the $\mathrm{N}$ terminus, comprising $\sim 22$ aa (Tmod1 residues 164-186), is critical for Tmod-mediated capping, since it covers the pointed end of the second subunit of the filament through interactions with actin subdomain 2 (Supplementary Fig. 4). Mutations in this region markedly reduce pointed-end capping ${ }^{7}$. Therefore, together with ABS1 and TMBS2, Lmods also appear to have lost this important capping element of ABS2.

While the divergent regions of ABS2 interact exclusively with the second actin subunit of the filament, the more highly conserved middle portion of the domain interacts at the interface between the first three subunits of the filament ${ }^{7}$. Therefore, one way in which Lmods could have acquired nucleation activity is by reinforcing these interactions. To test this hypothesis we designed Tmod-Lmod ABS2 hybrid constructs aimed at combining their respective pointed-end-capping and nucleating activities (Fig. 2a). The first two constructs ( $\mathrm{TL} 1_{\mathrm{ABS} 2}$ and $\mathrm{TL} 2_{\mathrm{ABS} 2}$ ) consisted of Tmod1 residues 164-229 and Lmod1 residues 365-486 (or Lmod 2 residues $246-367$ ). Strikingly, at $25 \mathrm{nM}$ both $\mathrm{TL} 1_{\mathrm{ABS} 2}$ and TL2 ${ }_{\mathrm{ABS} 2}$ had nucleation activities comparable to that of their full-length Lmod counterparts, albeit at higher concentrations the full-length proteins displayed higher activities (Fig. 3a). Importantly, both $\mathrm{TL} 1_{\mathrm{ABS} 2}$ and $\mathrm{TL} 2_{\mathrm{ABS} 2}$ had much higher activity than the wild-type ABS2 domains from which they originate (Fig. 3a). In the third construct (Tmod $1_{\mathrm{ABS} 2}$ Mut, Fig. 2a), 11 residues of $T$ mod $1_{\mathrm{ABS} 2}$ predicted to fall at the interface between three actin subunits in the filament were replaced by the corresponding residues in Lmod1, whose ABS2 has stronger nucleation activity than that of Lmod2. The 11 positions mutated were selected such that they were conserved within the separate Lmod and Tmod subfamilies, but not across subfamilies (Fig. 2a and Supplementary Fig. 1). Remarkably, contrary to Tmod1 $1_{\mathrm{ABS} 2}$, Tmod $11_{\mathrm{ABS} 2}$ Mut had strong nucleation activity that fell in between those of $\mathrm{TL} 1_{\mathrm{ABS} 2}$ and $\mathrm{TL} 2_{\mathrm{ABS} 2}$, and was much higher than that of Lmod $1_{\text {ABS2 }}$ (Fig. 3a).

We previously found that $T \bmod 1_{\mathrm{ABS} 2}$ binds a single actin monomer stabilized with gelsolin segment 1 (GS1) with micromolar affinity ${ }^{7}$. However, because filament nucleators are distinguished by their ability to recruit two or more actin subunits to form a nucleus, we asked whether the ABS2 variants displaying nucleation activity would bind two or more subunits. To test this idea, we used ITC. To prevent actin polymerization in these experiments, we used either LatB or GS1, both of which form 1:1 complexes with actin that cannot add along the long pitch helix of the actin filament (that is, binding of more than two actin subunits under these conditions is unlikely). Each experiment was fitted to either a one-site $(N=1)$ or a two-independent-site $(N=2)$ binding model, and the best model was chosen based on the $\chi^{2}$ value. As before ${ }^{7}$, the titration of Tmod1 $1_{\mathrm{ABS} 2}$ into actin (GS1) fitted equally well to both models, and thus the simplest solution $(N=1)$ was sufficient to explain this interaction (Supplementary Fig. 5a). The affinity of the interaction was relatively low, $K_{\mathrm{D}}=56 \pm 5 \mu \mathrm{M}$ (when $N$ is included in fitting). This experiment could not be performed with LatB-actin, because the complex precipitated in the ITC cell. In contrast to Tmod $1_{\mathrm{ABS} 2}$, all the ABS2 constructs with nucleation activity bound two actin subunits with either LatB or GS1 (Fig. 3b and Supplementary Figs 5 and 6$)$. ( $\operatorname{Lmod} 1_{\mathrm{ABS} 2}$ precipitated during the experiment and could not be analysed.) Invariably, the first binding site had nanomolar affinity, whereas the affinity of the second site was in the micromolar range. Note, however, that the affinities obtained with GS1-actin were always somewhat lower than those measured with LatB-actin, suggesting that GS1 allosterically lowers the affinity of ABS2 for actin. More generally, any factor preventing actin polymerization, including low salt, could in principle affect binding to monomeric actin. Therefore, rather than the exact affinity values, what these experiments tell us is that capping versus nucleation by ABS2 depends on whether this domain binds one (Tmod) or more (Lmod) actin subunits.

Structural bases of filament nucleation by ABS2. The observations described above raised several questions that required high-resolution structural information to be addressed, including: (a) how do the functional differences between the ABS2 of Tmods and Lmods correlate with structural differences? (b) are the hybrid Tmod-Lmod constructs properly folded? and (c) how does the ABS2 of a nucleator interact with actin compared with that of Tmod1 (ref. 7)? Three high-resolution crystal structures help us address these questions.

The structure of $\operatorname{Lmod} 1_{\mathrm{ABS} 2}$, the strongest nucleator among the naturally occurring ABS2s analysed here (Figs 1d and 3a), was determined at $1.54 \AA$ resolution (Table 1 and Supplementary Fig. 7a), and shows residues Ala-314 to Gln-486. The structure superimposes well with that of Tmod $1_{\mathrm{ABS} 2}$ (refs 6,7), with a root mean squared deviation (r.m.s.d.) of $0.57 \AA$ for 131 equivalent $\mathrm{C} \alpha$ atoms (Fig. 4a and Supplementary Movie 1). The major differences occur at the $\mathrm{N}$ and $\mathrm{C}$ termini of the domain, consistent with the lower sequence conservation observed within these areas (Fig. 2a and Supplementary Fig. 1). The C-terminal helix is rotated $\sim 4^{\circ}$ compared with $\operatorname{Tmod} 1_{\mathrm{ABS} 2}$ (Fig. 4a). However, this difference does not necessarily correlate to function, since the C-terminal helix is generally flexible and changes conformation when bound to actin 7 . The differences at the $\mathrm{N}$ terminus appear to be more important. Indeed, the loop of Tmod1 implicated in pointed-end capping (Tyr-170 to Val-183) is missing in Lmods, and the corresponding region of Lmod1 visualized in the electron density map (Ala-314 to Phe-318) is oriented in the opposite direction to that of $T \bmod 1_{\mathrm{ABS} 2}$ in the unbound $^{6}$ as well as the actin-bound ${ }^{7}$ structures.

The structure of $\mathrm{TL} 1_{\mathrm{ABS} 2}$, the strongest of the hybrid nucleators (Fig. 3a), was determined at $2.1 \AA$ resolution (Table 1 and Supplementary Fig. 7b), and shows Tmod1 residues Glu-177 to Phe-229 followed by Lmod1 residues Ala-365 to Gln-486 (Fig. 4b and Supplementary Movie 2). The structure superimposes well with those of Tmod $1_{\mathrm{ABS} 2}$ (r.m.s.d. of $0.41 \AA$ for 125 equivalent $\mathrm{C} \alpha$ atoms) and $\operatorname{Lmod} 1_{\mathrm{ABS} 2}$ (r.m.s.d. of $0.27 \AA$ for 124 equivalent $\mathrm{C} \alpha$ atoms). What is more, the side chains in the hydrophobic core of $\mathrm{TL} 1_{\mathrm{ABS} 2}$ have the same rotamer orientations as in the two parent structures. Therefore, the strong nucleation activity of $\mathrm{TL}_{\mathrm{ABS} 2}$ is solely due to its amino-acid composition, and not to changes in the overall structure.

The structure of $\mathrm{TL} 1_{\mathrm{ABS} 2}$ bound to actin was determined by fusing this domain $\mathrm{C}$ terminally to GS1, using a 9-aa flexible linker in between the two proteins (GGSGGSGGS), as done previously for $\mathrm{Tmod} 1_{\mathrm{ABS} 2}$ (ref. 7 ). Since $\mathrm{TL} 1_{\mathrm{ABS} 2}$ binds at least two actins (Fig. 3b), crystallization trials were conducted with the 1:1 purified complex (see Methods), as well as with addition of excess GS1-actin or LatB-actin. However, crystals were only obtained for the 1:1 complex. The structure was determined at $2.4 \AA$ resolution (Table 1 and Supplementary Fig. 7c). Overall, the structure is very similar to that of $\operatorname{Tmod}_{\mathrm{ABS} 2}$-actin. However, when the actin portion of these two structures are superimposed, $\mathrm{TL} 1_{\mathrm{ABS} 2}$ appears shifted on the actin surface by $\sim 2.5 \AA$ compared with Tmod $1_{\mathrm{ABS} 2}$ (Fig. $4 \mathrm{c}$ and Supplementary Movie 3). As a consequence, when the structure of $\mathrm{TL} 1_{\mathrm{ABS} 2}$-actin is 


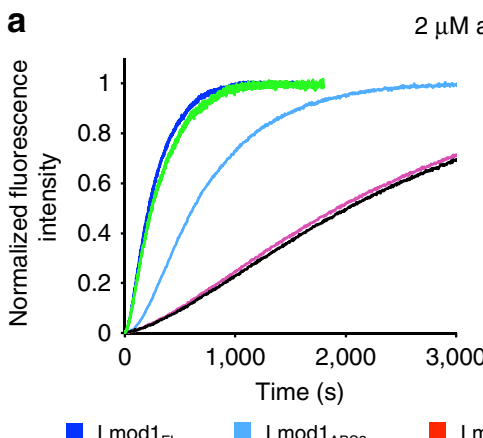

$2 \mu \mathrm{M}$ actin $(6 \%$ pyrene-labeled) $+25 \mathrm{nM} \mathrm{Lmod} \mathrm{(or} \mathrm{Tmod)}$
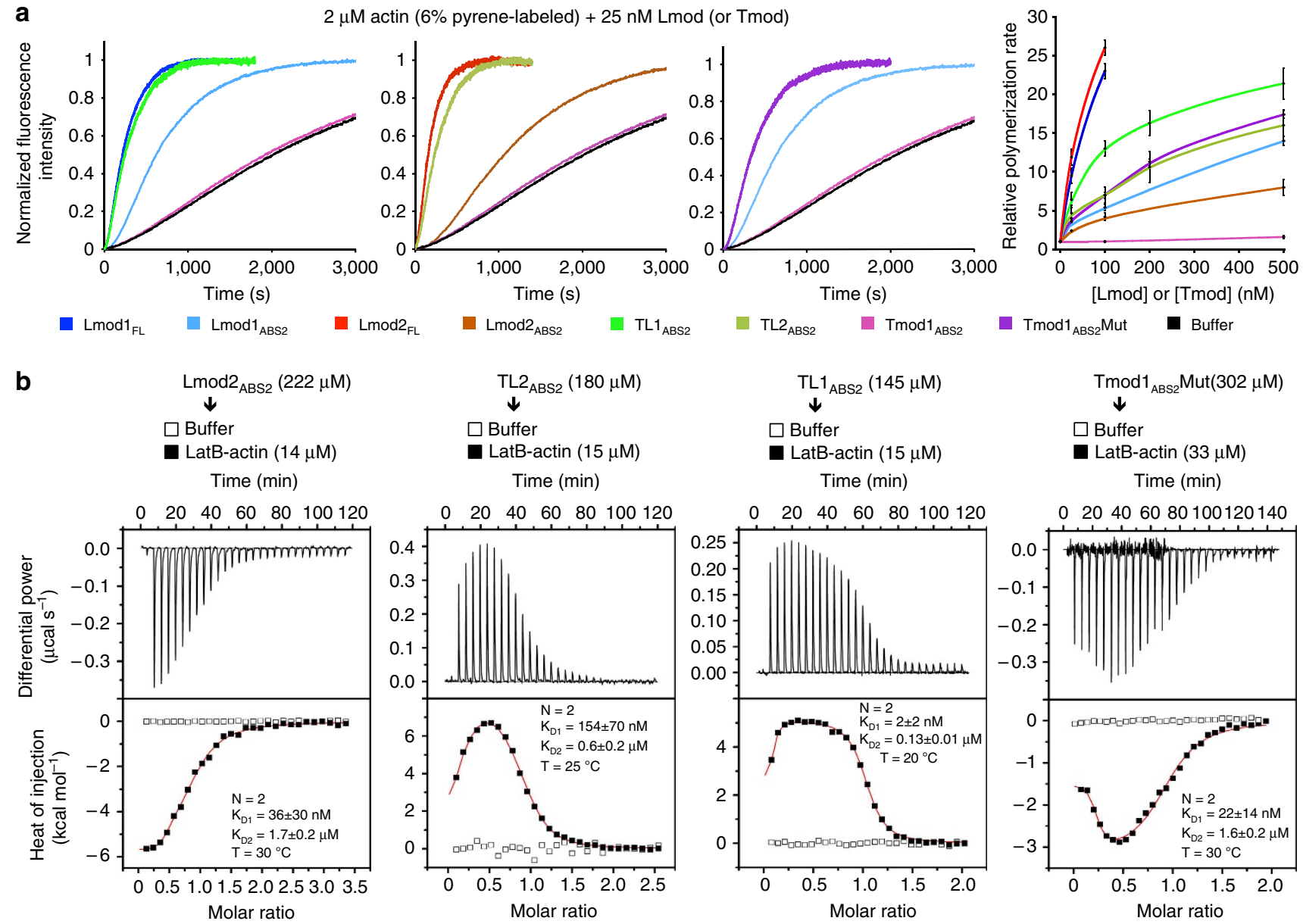

Figure 3 | Filament nucleation by ABS2. (a) Time courses of polymerization and concentration dependence of polymerization rates of ABS2 constructs (as indicated) compared with full-length Lmod1 and Lmod2 (colour coded). Experimental conditions listed on top. (b) ITC titrations of ABS2 constructs (as indicated) into LatB-actin. The experimental conditions and fitting parameters are listed with each experiment. All the titrations fitted to a two-binding-site model (see also Supplementary Figs 3 and 4). Open symbols correspond to titrations into buffer. Errors correspond to the s.d. of the fits.

superimposed onto the second actin subunit of the 3.7 ̊-resolution EM structure of the filament ${ }^{20}$, as proposed for Tmod1 $1_{\mathrm{ABS} 2}$ (ref. 7), this shift inserts $\mathrm{TL} 1_{\mathrm{ABS} 2}$ deeper into the groove formed at the interface between the three actin subunits (Fig. $4 \mathrm{~d}$ and Supplementary Movie 4). The residues of ABS2 that interact in this groove form part of the so-called 'ascending loops' of the LRR domain, which connect the $\beta$-strand and $\alpha$-helix of each repeat, and are commonly implicated in protein-protein interactions ${ }^{21}$. Strikingly these loops comprise several residues that are independently conserved among Tmods and Lmods, but not across the two subfamilies (Fig. 2a and Supplementary Figs 1 and 4). As shown above, replacing 11 such residues in Tmod1 $1_{\text {ABS2 }}$ by their Lmod1 counterparts was sufficient to produce the powerful nucleator $\operatorname{Tmod}_{1} 1_{\mathrm{ABS}} \mathrm{Mut}$ (Figs $3 \mathrm{a}$ and $4 \mathrm{e}$ ).

Role of Lmod and Tmod domains in cellular localization. The results described above suggest that Lmods have lost key elements needed for pointed-end capping and localization in Tmods, including $A B S 1, T M B S 2$ and the $N$ terminus of ABS2. On the other hand, we found that the nucleation activity of Lmods depends strongly on ABS2 (Fig. 3), but is additionally modulated by interaction of the N-terminal region with TM (Fig. 2b), which binds along the length of the actin filament. These observations prompted us to explore the role of the $\mathrm{N}$-terminal region and ABS2 in localization of Lmod versus Tmod. For this, Lmod and Tmod constructs were expressed in neonatal rat cardiomyocytes.
Co-expression of $\mathrm{Tmod}_{\mathrm{FL}}$-green fluorescent protein (GFP) with either $T \bmod 1_{\mathrm{ABS} 2}-\mathrm{mCherry}$ or $\operatorname{Tmod} 1_{\mathrm{N}}-\mathrm{mCherry}$ confirmed that both the $\mathrm{N}$ - and C-terminal capping domains ${ }^{4,22}$ are required for proper pointed-end localization of Tmod1 (Fig. 5a-d). As expected, Tmod1 $1_{\mathrm{FL}}$ localized to filament pointed ends near the M-line marker myomesin (Supplementary Fig. 8a), and well segregated from the Z-line marker $\alpha$-actinin (Fig. 5c). Both Tmod1 $1_{\mathrm{FL}}$ and $\alpha$-actinin showed well-defined periodicity in their average fast Fourier transform power spectra, with a power peak frequency of $0.572 \mu \mathrm{m}^{-1}$, corresponding to a distance of $1.75 \mu \mathrm{m}$ between $\mathrm{M}$ lines or $\mathrm{Z}$ lines, respectively (Fig. $5 \mathrm{~d}$ ). In contrast, $T \bmod 1_{\mathrm{ABS} 2}$ and $T \bmod 1_{\mathrm{N}}$ showed more diffuse localization along myofibrils, characterized by the loss of periodicity in their power spectra. This is consistent with the notion that the N-terminal region and ABS2 are both required for pointed-end capping by $\mathrm{Tmod}^{4}$, which is specifically accomplished by interactions of ABS1 and the N-terminal portion of ABS2 with the pointed ends of the first and second subunits of the filament, respectively.

In light of these results, our analysis of Lmod2 revealed a few surprises. As we previously reported ${ }^{11,19}, \operatorname{Lmod} 2_{\mathrm{FL}}$ was enriched near $\mathrm{M}$ lines (Fig. 5e,h and Supplementary Fig. 8b), somewhat similar to Tmod1 $1_{\mathrm{FL}}$. However, contrary to $T \bmod 1_{\mathrm{FL}}, \operatorname{Lmod} 2_{\mathrm{FL}}$ also showed diffuse localization along the length of the actin filaments in sarcomeres, with the exclusion of $\mathrm{Z}$ lines. Furthermore, while in the average power spectrum $\operatorname{Lmod} 2_{\mathrm{FL}}$ 
Table 1 | Crystallographic data and refinement statistics.

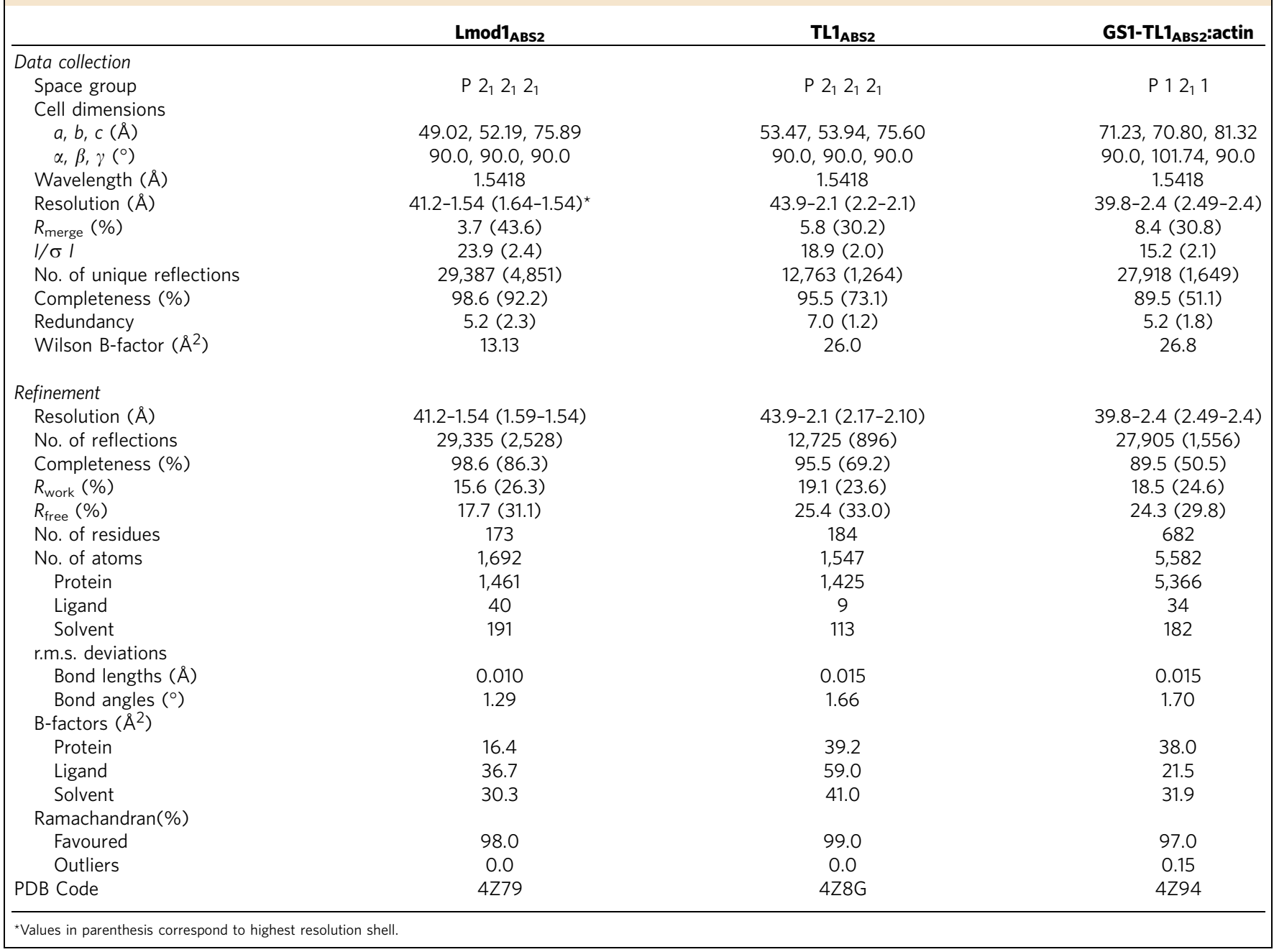

displayed clear periodicity (Fig. 5h), line scans showed that its distribution was broader than that of $\operatorname{Tmod}_{\mathrm{FL}}$ (compare Fig. $5 \mathrm{c}, \mathrm{g})$. Lmod $2_{\mathrm{ABS} 2}$ displayed relatively uniform localization along the length of the actin filaments, except $\mathrm{Z}$ lines, but unlike Lmod2 $2_{\mathrm{FL}}$ was not enriched near $\mathrm{M}$ lines (Fig. 5e,g). More surprising, however, was the finding that $\operatorname{Lmod} 2_{\mathrm{N}}$, which lacks ABS1 and TMBS2 (Fig. 2a,c), was enriched near $M$ lines (Fig. 5f,g). Unlike Tmod $1_{\mathrm{ABS} 2}$ and $T \bmod 1_{\mathrm{N}}$, the power spectra of both $\operatorname{Lmod} 2_{\mathrm{ABS} 2}$ and $\operatorname{Lmod} 2_{\mathrm{N}}$ displayed clear periodicity, with power peaks at 0.59 and $0.55 \mu \mathrm{m}^{-1}$, respectively (Fig. $5 \mathrm{~h}$ ). It thus appears that the $\mathrm{N}$-terminal region is important for $\operatorname{Lmod} 2_{\mathrm{FL}}$ enrichment near $M$ lines, whereas ABS2 drives its localization along the length of the actin filaments. Because Lmod $2_{\mathrm{N}}$ lacks ABS1 and TMBS2, its localization near pointed ends must depend on other interactions, possibly involving TMBS1 and the negatively charged sequence replacing TMBS2 (Supplementary Fig. 1). On the other hand, the lack of a pointed-end-binding sequence at the $\mathrm{N}$ terminus of ABS2 (Fig. 2a) allows it to bind in every groove between three actin subunits along the entire length of the filament (Fig. 4d). Tmod's ABS2 cannot do this, because it interacts mostly with a single actin subunit (Supplementary Fig. 5a) and has a pointed end directing sequence at the $\mathrm{N}$ terminus ${ }^{7}$.

Lmod1 differs substantially from Lmod2 (Fig. 1a and Supplementary Fig. 1a) and is predominantly expressed in smooth muscle cells $s^{8,9,12}$. Therefore, while here we exogenously expressed Lmod1 in cardiomyocytes, these results must be viewed as preliminary. Both $\operatorname{Lmod} 1_{\mathrm{FL}}$ and $\operatorname{Lmod}_{\mathrm{N}}$ unexpectedly localized near $\mathrm{Z}$ lines, forming a double band on both sides from $\alpha$-actinin, and were also slightly enriched near $M$ lines (Supplementary Fig. 9). Lmod1 $1_{\mathrm{ABS} 2}$ localized uniformly along myofibrils, without apparent periodicity. Our interpretation of these results is that cardiomyocytes lack smooth muscle-specific interactions necessary for $\operatorname{Lmod} 1_{\mathrm{FL}}$ and $\operatorname{Lmod} 1_{\mathrm{N}}$ enrichment near pointed ends, whereas the closely related ABS2s of Lmod1 and Lmod2 share the ability to bind along the length of the actin filament.

Tmod does not compete with Lmod during nucleation. We have shown here that Tmod and Lmod display similar but distinct localization in muscle sarcomeres. We have also shown that Lmod strongly nucleates actin polymerization, whereas Tmod does not. Finally, we have demonstrated that Lmod has lost key features required for pointed-end capping by Tmod, including ABS1, TMBS2 and the $\mathrm{N}$ terminus of ABS2. The question remains, does these two related families of proteins compete with each other for binding to the pointed end? In other words, does Lmod remain bound after nucleation to cap the pointed end the way Arp2/3 complex does? Pointed-end elongation/depolymerization is slow, such that any effect of Lmod on pointed-end kinetics is masked by its strong nucleation 
a

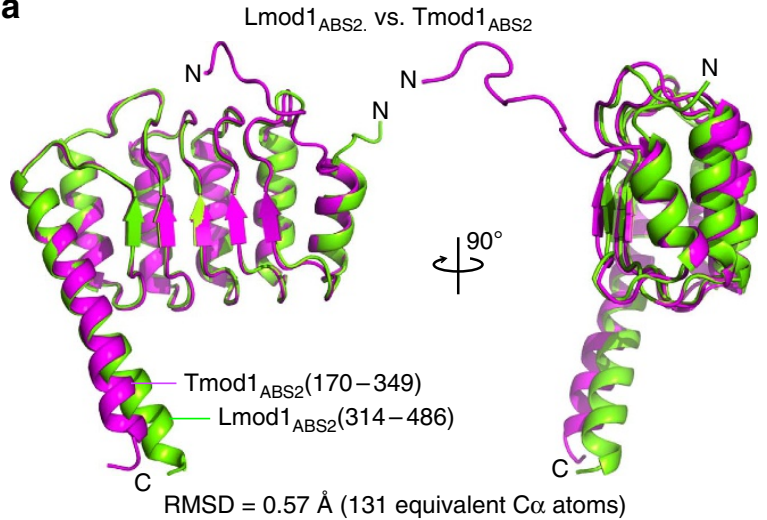

b

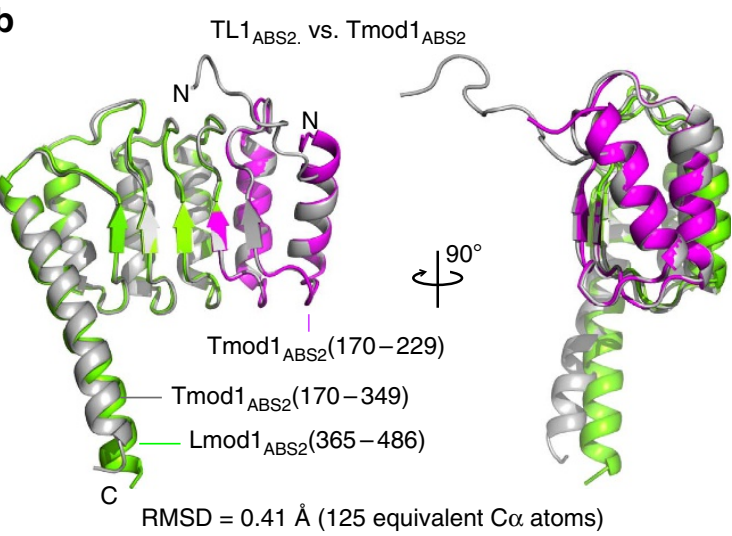

C

$\mathrm{TL}_{\mathrm{ABS}} \mathbf{a}^{- \text {actin vs. }} \mathrm{Tmod}_{\mathrm{ABS2}}{ }^{- \text {actin }}$

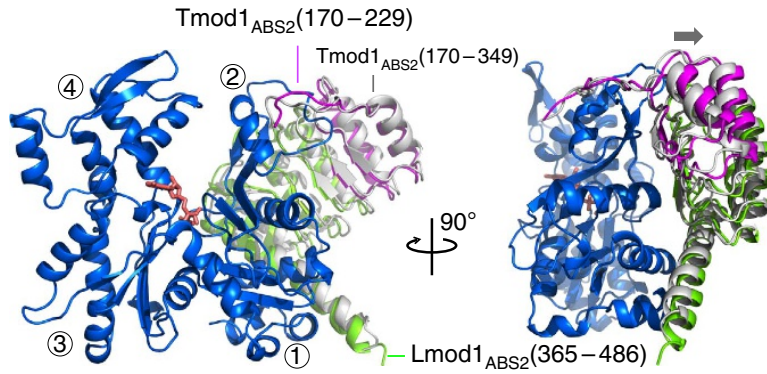

RMSD (actin to actin) $=0.58 \AA$ (300 equivalent $\mathrm{C} \alpha$ atoms)

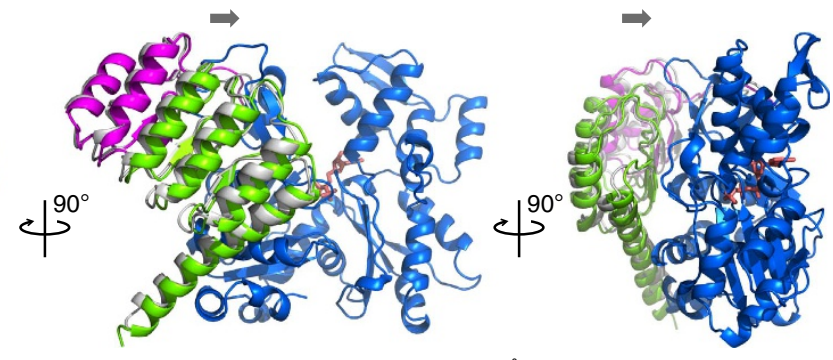

$\operatorname{RMSD}\left(\operatorname{Tmod}_{\mathrm{ABS} 2}\right.$ to $\left.\mathrm{TL} 1_{\mathrm{ABS} 2}\right)=0.45 \AA$ (129 equivalent $\mathrm{C} \alpha$ atoms)

d
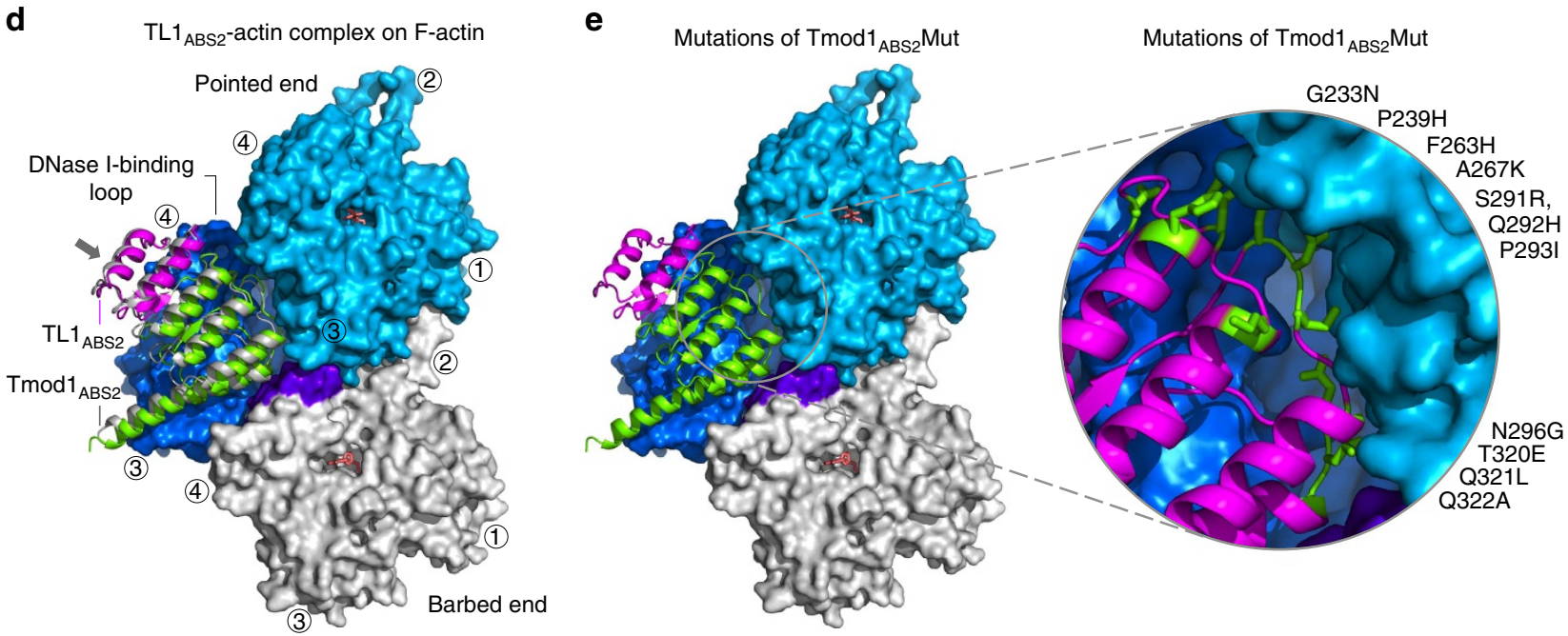

Figure 4 | Structures of ABS2 constructs alone and bound to actin. (a) Superimposition of the structures of $\operatorname{Tmod1}_{\mathrm{ABS2}}\left(\operatorname{magenta}^{7}\right.$ and $\mathrm{Lmod1}_{\mathrm{ABS} 2}$ (green), showing two orientations $90^{\circ}$ apart (see Supplementary Movie 1). Note that the structures superimpose well overall, except for the $\mathrm{N}$ and $\mathrm{C}$ termini (the r.m.s.d. for equivalent $\mathrm{C} \alpha$ is indicated). (b) Superimposition of the structures of $\mathrm{Tmod}_{\mathrm{ABS2}}$ (grey) and TL1 $1_{\mathrm{ABS} 2}$ (magenta and green, according to Fig. 2a) (see Supplementary Movie 2). (c) Superimposition of the structures of complexes of actin (blue) with the hybrid constructs GS1-Tmod1 ABS2 $_{2}$ $(\text { grey })^{7}$ and GS1-TL1 $1_{\mathrm{ABS} 2}$ (magenta and green), showing four orientations $90^{\circ}$ apart (see Supplementary Movie 3 ). GS1 is not shown, and actin is only shown for the complex with GS1-TL1 $1_{\mathrm{ABS} 2}$. Arrows indicate a slight shift of $\mathrm{TL1}_{\mathrm{ABS2}}$ on actin compared with $\mathrm{Tmod}_{\mathrm{ABS2}}$. (d) Superimposition of the complexes of actin with $\mathrm{TL1}_{\mathrm{ABS2}}$ (magenta and green) and $\operatorname{Tmod1}_{\mathrm{ABS} 2}$ (grey) onto the second actin subunit at the pointed end of the actin filament 7,20 . Only three subunits of the filament are shown (marine, blue, and grey-purple). ABS2 contacts all three subunits. The arrow indicates a slight shift of TL1 ${ }_{A B S 2}$ compared with $\operatorname{Tmod1}_{\mathrm{ABS} 2}$ that inserts it deeper into the groove formed at the interface between actin subunits. (e) Same as (d) but showing a close-up view of the location of the 11 residues of $\operatorname{Tmod1}_{\mathrm{ABS} 2}$ that were mutated to their Lmod1 counterparts in construct $\operatorname{Tmod1}_{\mathrm{ABS} 2}$ Mut.

activity in bulk or total internal reflection fluorescence (TIRF) assays. Therefore, to address this question we tested whether nucleation by Lmod was in any way affected by the presence of Tmod. Yet, we found that even a fourfold excess of Tmod1 had no effect on the nucleation activity of Lmod2 alone or in the presence of TM (Fig. 6a,b). These results allow us to conclude that Tmod1 and Lmod2, which are both expressed in sarcomeres ${ }^{1,11}$, do not compete biochemically with each other. Tmod binds with very high affinity to the pointed end, particularly in the presence of $\mathrm{TM}^{7,17}$, and it would be expected to cap the pointed end of the 
a

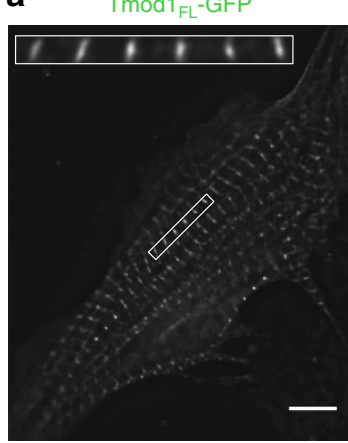

b

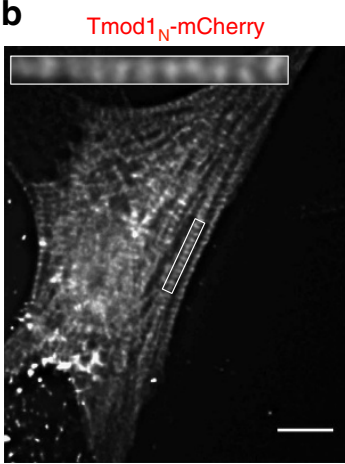

e $\quad L \bmod 2_{F L}-G F P$

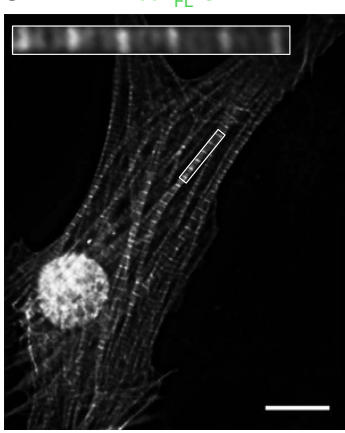

f

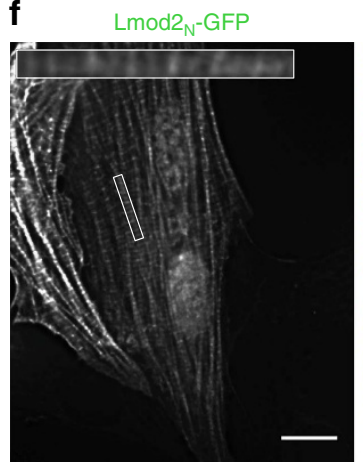

anti- $\alpha$-actinin

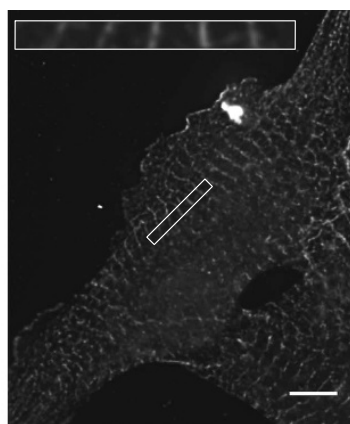

Merge

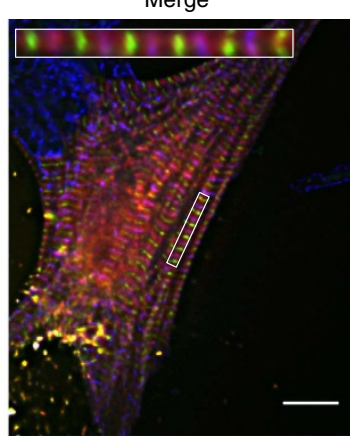

anti- $\alpha$-actinin
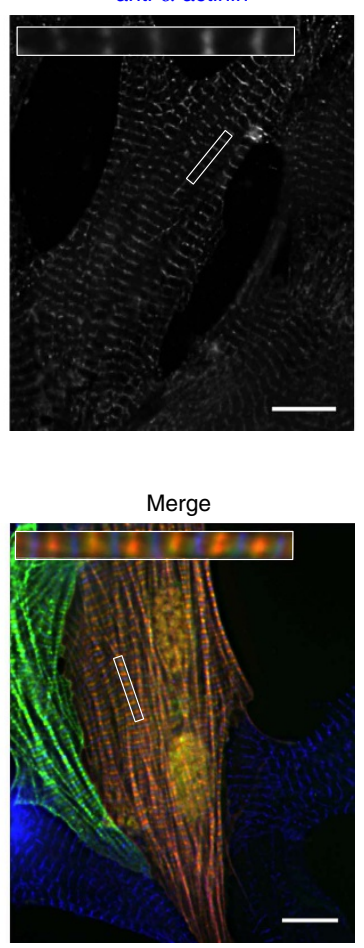

Tmod1 1 -mCherry

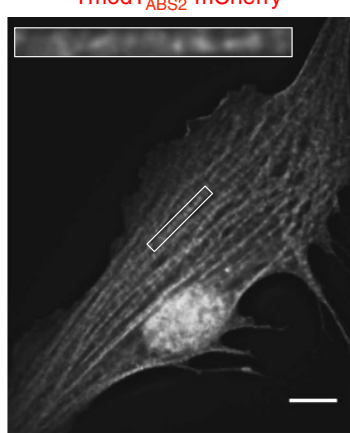

- Tmod1 $1_{\mathrm{FL}} \quad \alpha$-actinin

C
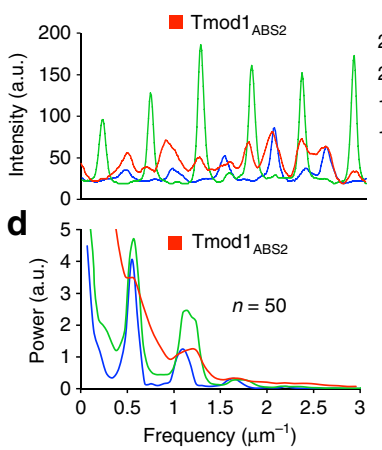

Lmod2 ${ }_{\mathrm{ABS2}}$-mCherry
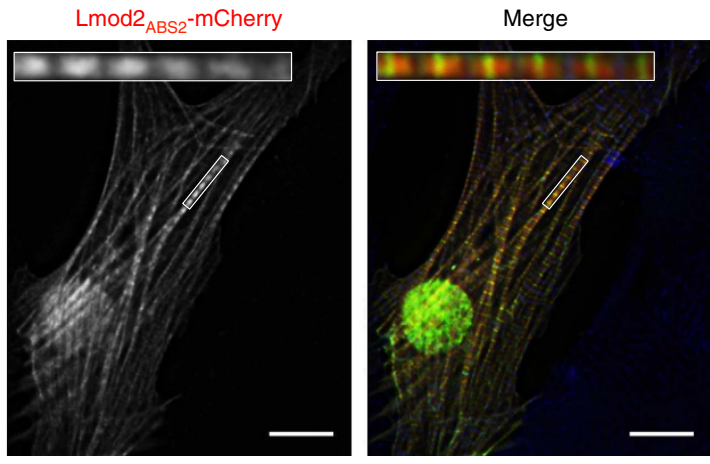

- $\alpha$-actinin
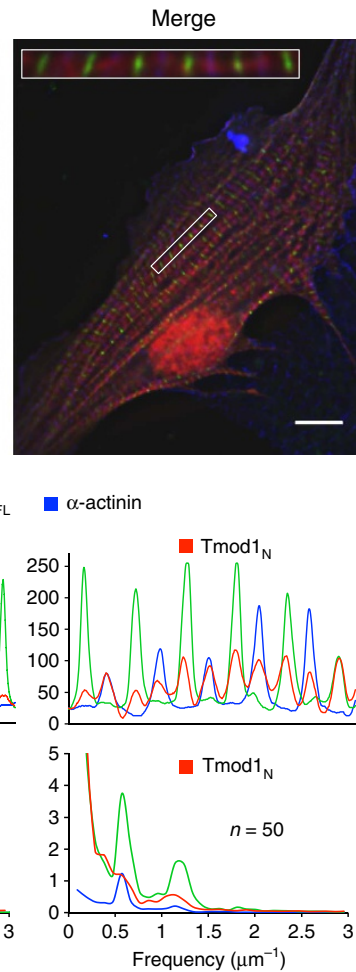

Merge

g
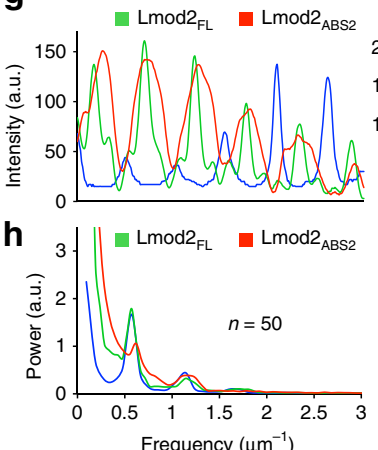
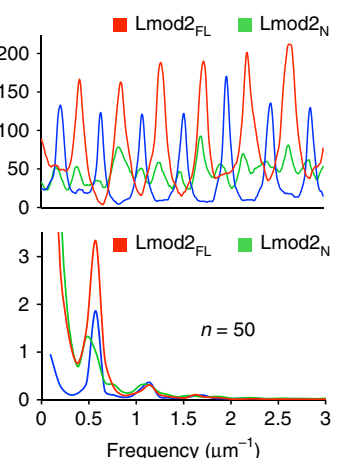

Figure 5 | Localization of Tmod1 and Lmod2 constructs in sarcomeres. (a,b) Cardiomyocytes co-transfected on day 1 with Tmod1 $\mathrm{FL}^{-G F P}$ and $\operatorname{Tmod1}_{\mathrm{ABS} 2}-\mathrm{mCherry}$ (or Tmod1 $1_{\mathrm{N}}-\mathrm{mCherry}$ ), fixed $24 \mathrm{~h}$ after transfection, and stained with anti- $\alpha$-actinin antibodies (Z-line marker). Note that $\operatorname{Tmod1}_{\mathrm{FL}}$-GFP and $\alpha$-actinin are shown separately only in part (a) but these markers are also present in part. (b). (c) Line-scans of each marker (colour coded) along a representative myofibril (insets in parts $\mathbf{a}, \mathbf{b}$ ). (d) Average power spectra resulting from one-dimensional (1D) fast Fourier transform (FFT) analysis of 50 line-scans from six cells transfected with $\operatorname{Tmod1}_{\mathrm{ABS} 2}$ (or $\operatorname{Tmod}_{\mathrm{N}}$ ). The frequency of the power peak for $\operatorname{Tmod1}_{\mathrm{FL}}$ and $\alpha$-actinin is $0.572 \mu \mathrm{m}{ }^{-1}$, corresponding to a distance of $1.75 \mu \mathrm{m}$ between $\mathrm{M}$ lines or $Z$ lines, respectively. In contrast, there is no defined power peak in the spectra of Tmod1 ${ }_{\mathrm{ABS} 2}$ or Tmod1 $1_{\mathrm{N}}$, reflecting a loss of periodicity in their localization. (e,f) Cardiomyocytes co-transfected with Lmod2 $2_{\mathrm{FL}}-\mathrm{GFP}$ and Lmod2 $2_{\mathrm{ABS} 2}-\mathrm{mCherry}$ (or Lmod $2_{\mathrm{FL}}-\mathrm{mCherry}$ and $\mathrm{Lmod} 2_{\mathrm{N}}-\mathrm{GFP}$ ) and stained with anti- $\alpha$-actinin antibodies. (g) Line-scans of each marker (colour coded) along a representative myofibril (insets in parts $\mathbf{e , f}$ ). (h) Average power spectra resulting from 1D FFT analysis of 50 line-scans from six cells transfected with Lmod2 $2_{\text {ABS2 }}$ (or $L \bmod 2_{N}$ ). All the spectra show similar periodicity, with power peaks at 0.59 and $0.55 \mu \mathrm{m}^{-1}$ for $\operatorname{Lmod} 2_{\mathrm{ABS} 2}$ and $\operatorname{Lmod} 2_{\mathrm{N}}$, respectively. The Tmod1 and Lmod2 constructs are defined in Methods and in Fig. 1a. Scale bars, $10 \mu \mathrm{m}$. 


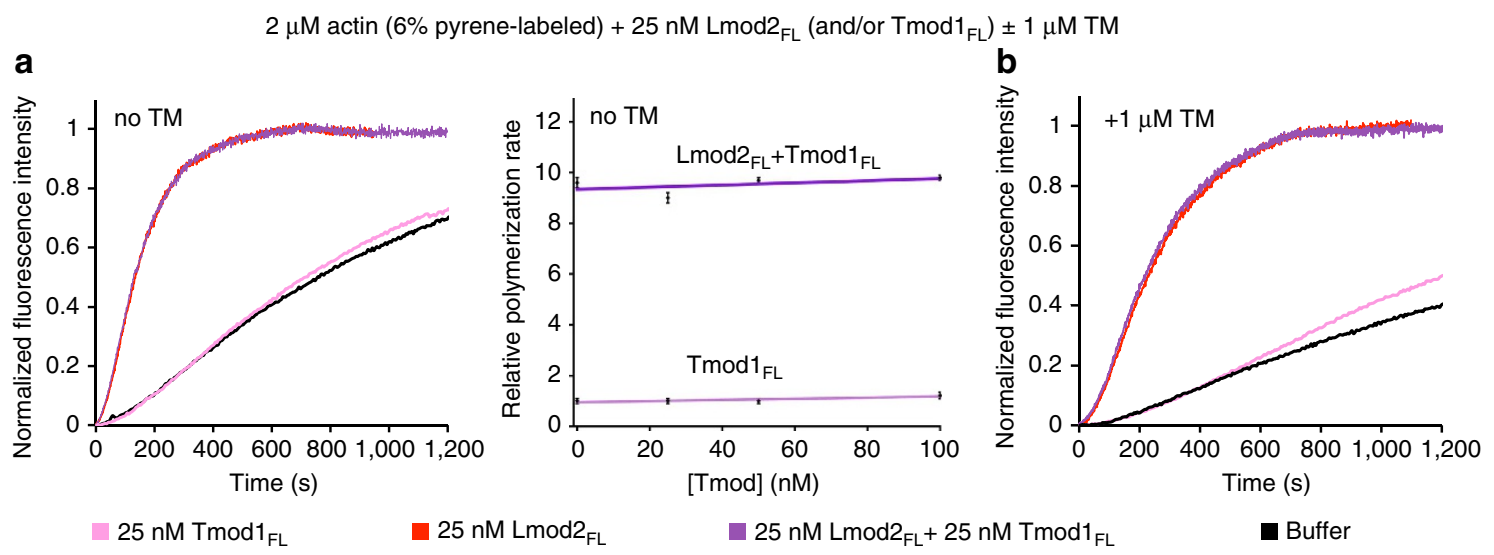

Figure 6 | Tmod1 does not compete with Lmod2 during nucleation with or without TM. (a) Time course of polymerization and concentration dependence of polymerization rates by $L \operatorname{Lmod} 2_{\mathrm{FL}}$ in the presence of increasing concentrations of $\mathrm{Tmod}_{\mathrm{FL}}$. Experimental conditions listed on top. The concentration dependence is displayed as the mean of three experiments \pm s.e.m. (b) Time course of polymerization by $\mathrm{Lmod}_{\mathrm{FL}}$ in the presence of Tmod1 $\mathrm{FL}_{\mathrm{L}}$ and $\mathrm{TM}$.

Lmod-nucleated filaments. Therefore, if Lmod remained bound to the pointed end after nucleation it should be released by Tmod, thus increasing the amount of Lmod available for nucleation. The fact that bulk polymerization does not increase with the addition of Tmod strongly suggests that Lmod does not stay bound at the pointed end after nucleation.

\section{Discussion}

By studying Tmod and Lmod in parallel, we have established here how these two related subfamilies of proteins have evolved different functions-pointed-end capping and filament nucleation, respectively. We showed that Tmod and Lmod do not compete biochemically with each other, and display similar but distinct localization in muscle sarcomeres (Figs 5 and 6). Rather unexpectedly, we found that the C-terminal extension of Lmods, which distinguishes them from Tmods, was not the main source of the functional differences between the two subfamilies. Thus, Tmod failed to gain strong nucleation activity even after addition of the C-terminal extension of Lmod, whereas Lmod retained significant nucleation activity after removal of this extension. This was true for both Lmod 2 and the smooth muscle isoform Lmod1, characterized here for the first time. It thus appears that in Lmod the WH2 domain plays only an auxiliary role in nucleation, possibly by helping to recruit the third actin subunit of the polymerization seed, which also interacts with ABS2 and the first two actin subunits (Fig. 4d).

The emergence of ABS2 as the main source of the differences between Tmod and Lmod was unexpected, since this domain corresponds to the most highly conserved region between these two subfamilies. The transition from capping to nucleation within this domain involves two major adaptations. First, in Lmod ABS2 lacks the $\mathrm{N}$-terminal region that in Tmod interacts with the DNase I-binding loop at the pointed end of the second actin subunit of the filament ${ }^{7}$. In this way, Lmod has lost a major determinant of pointed-end capping, which would put it in competition with Tmod in vitro and in cells. Second, the ascending loops of the LRR domain have different sequence in Lmod compared with Tmod. These loops bind in the groove formed at the interface between three actin subunits of the filament. We have shown here that it is sufficient to mutate 11 amino acids within these loops to turn Tmod's ABS2 into a powerful nucleator. This is achieved by increasing the affinity of this domain for two or more actin subunits, which would interfere with Tmod's capping activity in cells. Curiously, the hybrid constructs combining the capping $\mathrm{N}$ terminus of Tmod1's ABS2 with the added affinity of Lmod's ABS2 were better nucleators than those of the wild-type proteins. This surprising result can now be rationalized. The ABS2 of Lmod has the required affinity to recruit two or more subunits for nucleation, but by adding a pointed-end-binding element, we have limited the ability of this domain to bind along the length of the filament when in isolation (Fig. 5), that is, the hybrid constructs emphasize nucleation at the expense of filament side-binding. These constructs constitute the smallest single-domain protein with strong nucleation activity identified thus far.

Several adaptations of Lmod for nucleation occur within the $\mathrm{N}$-terminal region, including the lack of ABS1 and TMBS2 necessary for pointed end capping by Tmod. Despite these changes, however, the N-terminal region of Lmod2 localizes near M-lines on its own, whereas that of Tmod does not (Fig. 5). TM binding through TMBS1 is probably not sufficient to explain this difference, since this site is conserved in both Lmod1 and Tmod1. The sequence between TMBS1 and ABS2 is very different in Tmod1, Lmod1 and Lmod2, and probably holds the key to understand their different localizations, which will be the subject of future investigation. Finally, Lmod's N-terminal region participates in nucleation through interaction with TM, which upregulates this activity, a fact that we had previously observed with Lmod2 (refs 11,19) and is confirmed here for Lmod1.

In summary, nucleation in Lmod is the result of two major adaptations - the loss of capping elements present in Tmod and the specialization of ABS2 for recruitment of two or more actin subunits. The N-terminal region is necessary for localization, and modulates the nucleation activity through interaction with TM, whereas the $\mathrm{WH} 2$ domain-containing C-terminal extension adds to the overall nucleation activity, likely by securing the binding of the third actin subunits of the nucleus.

\section{Methods}

Proteins. The exact amino-acid composition of all the proteins, fragments and hybrid constructs used in this study are schematically depicted in the main figures of the paper (Figs 1 and 2a), whereas all the primers used in cloning are listed in Supplementary Table 1.

Since our original characterization of human cardiac Lmod2 (ref. 11), the sequence has been corrected (UniProt: Q6P5Q4-1). The originally deposited sequence contained two large deletions of 32 aa (residues 99-130) and 20 aa (residues 428-447) within the N- and C-terminal regions, respectively. To build the correct sequence, the missing amino acids were added by PCR to an incomplete clone (IHS1382-8558565) previously purchased from Open Biosystems. The primers coding for the missing amino acids also introduced silent EcoRI and XhoI sites that were subsequently used for ligation of the amplified fragments. The cDNA encoding for human Lmod1 (UniProt: P29536-1) was purchased from Open Biosystems.

Most proteins expressed here were cloned between the NdeI and SapI sites of vector pTYB1 (NEB). The ABS1 fragments were cloned between the SapI and XhoI 
sites of vector pTYB11 (NEB). These vectors comprise a chitin-binding domain for affinity purification and an intein domain for self-cleavage of the affinity tag after purification. Because full-length Lmods are highly susceptible to degradation, a second affinity purification tag (a His-tag) was added at the $\mathrm{N}$ terminus during cloning. The double affinity purification allows separating the full-length protein from the degradation fragments. The cDNA encoding for human Tmod1 (UniProt: P28289-1) and human gelsolin (UniProt: P06396-2) were purchased from American Type Culture Collection. The Tmod1 fragments were cloned into vector pTYB1 as described above. The 11 mutations of construct Tmod1 $1_{\mathrm{ABS} 2} \mathrm{Mut}$ were generated using the QuickChange mutagenesis kit (Stratagene). The hybrid construct Tmod1-Lmod2 $2_{C}$ was obtained by introducing silent mutations at the junction between the two proteins using the forward and reverse primers, such as to produce a new MfeI restriction site that was then used for ligation. The hybrid constructs $T L 1_{\mathrm{ABS} 2}$ and $\mathrm{TL} 2_{\mathrm{ABS} 2}$ were obtained by amplifying fragments:

$\operatorname{Tmod} 1_{164-229}$, $\operatorname{Lmod} 1_{365-486}$ and $\operatorname{Lmod} 2_{246-367}$ with primers introducing silent NruI restriction sites at the junctions. The hybrid constructs GS1-TL1 $1_{\mathrm{ABS} 2}$ was obtained as described above, using as a template our previously published GS1-Tmod1 $1_{\mathrm{ABS} 2}$ (ref. 7) and ligating Lmod1 ${ }_{365-486}$ after Tmod1 residue Phe-229. All the ligation products were cloned between the NheI and SapI sites of vector pTYB1. Human gelsolin segment 1 (GS1, residues 1-125, UniProt: P06396-2) was cloned between the NdeI and EcoRI sites of vector pTYB12 (NEB). Human profilin 1 (UniProt: P07737) was cloned between the SapI and EcoRI sites of vector pTYB11 (NEB).

All the proteins were expressed in BL21(DE3) cells (Invitrogen), grown in Terrific Broth medium at $37^{\circ} \mathrm{C}$ until the $\mathrm{OD}_{600}$ reached a value of $1.5-2$, followed by $16 \mathrm{~h}$ at $20^{\circ} \mathrm{C}$ in the presence of $0.5 \mathrm{mM}$ isopropyl- $\beta$-D-thiogalactoside. Cells were collected by centrifugation, re-suspended in $20 \mathrm{mM}$ HEPES pH 7.5, $500 \mathrm{mM}$ $\mathrm{NaCl}, 1 \mathrm{mM}$ EDTA and $100 \mu \mathrm{M}$ phenylmethyl sulfonyl fluoride and lysed using a microfluidizer (Microfluidics). All the proteins were first purified on a chitin affinity column according to the manufacturer's protocol (NEB). Full-length Lmods were additionally purified on a Ni-NTA column (Qiagen). Finally, all the proteins were purified by gel filtration on a SD200HL 26/600 column (GE) in $20 \mathrm{mM}$ HEPES (pH 7.5) and $200 \mathrm{mM} \mathrm{NaCl}$.

Arp2/3 complex was purified from bovine brain as we have described ${ }^{23}$. Briefly, frozen brain was homogenized in Arp buffer (20 mM HEPES ( $\mathrm{pH} 7.5), 100 \mathrm{mM}$ $\mathrm{KCl}, 1 \mathrm{mM} \mathrm{MgCl}$, $1 \mathrm{mM}$ EGTA, $1 \mathrm{mM}$ dithiothreitol (DTT)) supplemented with protease inhibitors and clarified by centrifugation at $12,000 \mathrm{~g}$ for $30 \mathrm{~min}$. The supernatant was loaded onto a Macro-Prep High Q column (Bio-Rad) pre-equilibrated with Arp buffer. The flow-through, containing Arp2/3 complex, was applied onto a WCA-affinity column equilibrated with Arp buffer. Arp2/3 complex was eluted in $20 \mathrm{mM}$ Tris ( $\mathrm{pH} 8.0$ ), $25 \mathrm{mM} \mathrm{KCl}, 400 \mathrm{mM} \mathrm{MgCl}_{2}, 1 \mathrm{mM}$ EGTA and $1 \mathrm{mM}$ DTT, concentrated and further purified through an SD200HL 26/ 600 column in Arp buffer. Actin ${ }^{24}$ and $\mathrm{TM}^{25}$ were purified as previously described. Briefly, actin was extracted from actomyosin acetone powder with G-buffer $(2 \mathrm{mM}$ Tris (pH 8.0), $0.2 \mathrm{mM} \mathrm{CaCl}_{2}, 0.2 \mathrm{mM} \mathrm{ATP,} 0.5 \mathrm{mM}$ DTT and $0.01 \% \mathrm{NaN}_{3}$ ), centrifuged at $20,000 \mathrm{~g}$ for $30 \mathrm{~min}$ and polymerized with addition of $50 \mathrm{mM} \mathrm{NaCl}$ and $2 \mathrm{mM} \mathrm{MgCl}$. The F-actin pellet was homogenized in G-buffer with the addition of $10 \mathrm{mM}$ DTT. After $1 \mathrm{~h}$, actin was dialysed exhaustively against G-buffer to remove DTT and then centrifuged for $45 \mathrm{~min}$ at $277,000 \mathrm{~g}$ to pellet any F-actin that did not depolymerize and any denatured actin. Tropomysin was extracted with $10 \mathrm{mM}$ Tris ( $\mathrm{pH} 8.0$ ), $0.5 \mathrm{mM} \beta$-mercaptoethanol from the solid fraction remaining after actin extraction from the acetone powder. The solution was clarified by centrifugation $\left(16,000 \mathrm{~g}\right.$ for $1 \mathrm{~h}$ ), purified by precipitation with $20 \% \mathrm{NH}_{4} \mathrm{SO}_{4}$ and centrifuged again. Tropomyosin was precipitated from the supernatant with $30 \%$ $\mathrm{NH}_{4} \mathrm{SO}_{4}$, dialysed against $2 \mathrm{mM}$ Imidazole, $5 \mathrm{mM} \beta$-mercaptoethanol and purified on a hydroxyapatite column with a linear gradient of $1-200 \mathrm{mM} \mathrm{KH}_{2} \mathrm{PO}_{4}(\mathrm{pH} 7.0)$, $1 \mathrm{M} \mathrm{KCl}$ and $2 \mathrm{mM}$ DTT.

The concentration of all the proteins was determined spectrophotometrically, using calculated extinction coefficients (Supplementary Table 2).

Actin polymerization assay. Actin polymerization was measured as the fluorescence increase resulting from the incorporation of pyrene-labelled actin into filaments, using a Cary Eclipse fluorescence spectrophotometer (Varian). Before data acquisition, $200 \mu \mathrm{Mg}$-ATP-actin at $2 \mu \mathrm{M}$ concentration (6\% pyrene labelled) was mixed with $5 \mu \mathrm{l}$ of Lmod or Tmod constructs in $10 \mathrm{mM}$ Tris (pH 8.0), $1 \mathrm{mM}$ $\mathrm{MgCl}_{2}, 50 \mathrm{mM} \mathrm{KCl}, 1 \mathrm{mM}$ EGTA, $0.2 \mathrm{mM}$ ATP, $0.5 \mathrm{mM}$ DTT and $0.1 \mathrm{mM} \mathrm{NaN}$. The final concentration of Lmod or Tmod in the polymerization reaction ranged between 5 and $500 \mathrm{nM}$ (as indicated in the figures). In some experiments, $1 \mu \mathrm{M} \mathrm{TM}$ was added to the actin before the addition of full-length Lmod1 or Lmod2, such that the total volume remained constant at $200 \mu \mathrm{l}$. Data acquisition started $10 \mathrm{~s}$ after mixing. All the measurements were done at $25^{\circ} \mathrm{C}$. Control experiments were carried out with addition of $5 \mu \mathrm{l}$ buffer. Relative polymerization rates were calculated as the maximal slope of a polymerization curve (between 0.1 and 0.4 of the maximum fluorescence) divided by the maximal slope of the actin control.

Isothermal titration calorimetry. ITC measurements were carried out on a VP-ITC apparatus (MicroCal). Samples were first dialysed for 2 days against $20 \mathrm{mM}$ HEPES (pH 7.5), $100 \mathrm{mM} \mathrm{NaCl}, 0.2 \mathrm{mM}$ ATP and $1 \mathrm{mM}$ DTT (ITC buffer). In experiments with LatB-actin, LatB was added to the buffer at a LatB:actin ratio of 1.2:1. In experiments with GS1-actin, actin and GS1 were mixed at a 1:1.5 molar ratio, and the complex was purified by gel filtration on a SD200HL 26/600 column (GE Healthcare) in ITC buffer.

Each experiment was carried out over a range of temperatures from $10^{\circ} \mathrm{C}$ to $30^{\circ} \mathrm{C}$, in $5^{\circ} \mathrm{C}$ increments, and the optimal temperature for each reaction is shown (Figs $2 \mathrm{c}$ and $3 \mathrm{~b}$ and Supplementary Figs 5 and 6). Titrations consisted of $10-\mu \mathrm{l}$ injections, lasting for 10 or $20 \mathrm{~s}$, with an interval of $4-5 \mathrm{~min}$ between injections. The concentration of the Lmod or Tmod titrant (listed in the figures) was 10- to 15-fold higher than that of actin in the cell of total volume $1.44 \mathrm{ml}$. The heat of binding was corrected for the heat of injection, determined by injecting titrant into buffer (open symbols in figures). Data were analysed using the program Origin (OriginLab Corporation).

Crystallization, data collection and structure determination. $L \bmod 1_{\mathrm{ABS} 2}$ at $10 \mathrm{mg} \mathrm{ml}^{-1}$ in $20 \mathrm{mM}$ HEPES (pH 7.5), $200 \mathrm{mM} \mathrm{NaCl}$ and $1 \mathrm{mM}$ DTT was crystallized at $20^{\circ} \mathrm{C}$ using the hanging drop method. The crystallization drop consisted of a 1:1 (v/v) mixture of protein solution and well solution $(100 \mathrm{mM}$ HEPES (pH 7.5), 10\% (v/v) isopropanol and 20\% (v/v) polyethylene glycol 3,350). For data collection, crystals were flash frozen in liquid nitrogen from a cryo-solution consisting of crystallization buffer with addition of $30 \%$ glycerol. $\mathrm{TL}_{\mathrm{ABS} 2}$ at $43 \mathrm{mg} \mathrm{ml}^{-1}$ in $20 \mathrm{mM}$ HEPES (pH 7.5), $300 \mathrm{mM} \mathrm{NaCl}$ and $1 \mathrm{mM}$ DTT was crystallized at $20^{\circ} \mathrm{C}$ using the sitting drop method. The crystallization drop consisted of a 1:1 (v/v) mixture of protein solution and well solution (100 mM MES (pH 6.0), $200 \mathrm{mM} \mathrm{LiSO}_{4}$ and 35\% (v/v) 2-methyl-2,4-pentanediol (MPD)). For data collection, crystals were flash frozen in liquid nitrogen directly from a drop.

The 1:1 complex of GS1-TL1 $1_{\mathrm{ABS} 2}$ :actin was purified by gel filtration on a SD200HL 26/600 column and concentrated to $12.5 \mathrm{mg} \mathrm{ml}^{-1}$ in $20 \mathrm{mM}$ HEPES (pH 7.5), $100 \mathrm{mM} \mathrm{NaCl}, 0.2 \mathrm{mM}$ ATP, $0.2 \mathrm{mM} \mathrm{CaCl}_{2}$ and $1 \mathrm{mM} \mathrm{DTT}$. Crystals were obtained at $20^{\circ} \mathrm{C}$ using the hanging drop method. The crystallization drop consisted of a 1:2 (v/v) mixture of protein solution and well solution $(100 \mathrm{mM}$ Tris $(\mathrm{pH} 8.8), 200 \mathrm{mM} \mathrm{LiSO}_{4}, 20 \%(\mathrm{v} / \mathrm{v})$ polyethylene glycol 3,350 and $15 \%(\mathrm{v} / \mathrm{v})$ glycerol). For data collection, crystals were flash frozen in liquid nitrogen from a cryo-solution consisting of crystallization buffer with $30 \%$ (v/v) glycerol.

$\mathrm{X}$-ray data sets were collected at $100 \mathrm{~K}$ using our home X-ray instrument Bruker X8 Prospector X-ray diffraction system, fitted with an I $\mu$ S microfocus sealed-tube X-ray source, Apex II CCD detector, 4-circle Kappa goniometer and an Oxford Cryostream 700 liquid nitrogen cooling system. The diffraction data sets were indexed and scaled with the Bruker program SAINT (version v8.18c). Molecular replacement solutions were obtained with the program Phenix ${ }^{26}$ using PDB entry 4PKI (our crystal structure of the GS1-Tmod1 $1_{\mathrm{ABS} 2}$ :actin complex ${ }^{7}$ ). Model building and refinement were carried out with the programs $\operatorname{Coot}^{27}$ and Phenix. Data collection and refinement statistics are listed in Table 1.

\section{Isolation of rat cardiomyocytes and cell transfection. Neonatal rat cardio-} myocytes were isolated and cultured as previously described ${ }^{28}$. Briefly, neonatal rat (Wistar Han) hearts were dissected and enzymatically digested with $0.5 \mathrm{mg} \mathrm{ml}^{-1}$ collagenase (Worthington Biochemical Corporation) and $0.6 \mathrm{mg} \mathrm{ml}^{-1}$ pancreatin (Gibco). After plating in culture medium (DMEM supplemented with 4:1 Medium 199 (Life Technologies), 10\% horse serum, 5\% heat inactivated fetal bovine serum, $4 \mathrm{mM}$ glutamine and $1 \%$ penicillin-streptomycin) for $\sim 70 \mathrm{~min}$ on uncoated plastic to discard fibroblasts, the cardiomyocytes were re-plated on collagen/fibronectincoated plastic dishes (Nunc). The culture media was exchanged after $24 \mathrm{~h}$ for maintenance media, consisting of $20 \%$ Medium 199, $4 \%$ horse serum, $4 \mathrm{mM}$ glutamine, $1 \%$ penicillin-streptomycin, $0.1 \mathrm{mM}$ phenylephrine, $0.01 \mathrm{mM}$ cytosine arabinoside and $75 \%$ DBSS-K $\left(6.8 \mathrm{gl}^{-1} \mathrm{NaCl}, 0.14 \mathrm{mM} \mathrm{NaH}_{2} \mathrm{PO}_{4}, 0.2 \mathrm{mM} \mathrm{CaCl}\right.$ $0.2 \mathrm{mM} \mathrm{MgSO}_{4}, 1 \mathrm{mM}$ dextrose and $2.7 \mathrm{mM} \mathrm{NaHCO}_{3}$ ). Cells were transiently transfected with EGFP and mCherry constructs $24 \mathrm{~h}$ after isolation using Escort III reagent (Sigma-Aldrich) according to the manufacturer's specifications and cultured in maintenance media until fixation. For expression in mammalian cells, Lmod and Tmod constructs, including Tmod1 $1_{\mathrm{N}}(1-163)$, Tmod $1_{\mathrm{ABS} 2}$ (164-351), $\operatorname{Lmod} 2_{\mathrm{N}}(1-179), \operatorname{Lmod} 2_{\mathrm{ABS} 2}(180-367), \operatorname{Lmod} 1_{\mathrm{N}}(1-298)$ and $\operatorname{Lmod} 1_{\mathrm{ABS} 2}$ (299-486), were cloned between the XhoI and BamHI sites of vector pEGFP-N1 (Clontech) or NheI and KpnI sites of vector mCherry2-N1 (Addgene plasmid \# 54517).

Immunofluorescence microscopy. Cells were fixed $24 \mathrm{~h}$ post-transfection with $4 \%$ paraformaldehyde for $20 \mathrm{~min}$ and permeabilized with $0.2 \%$ Triton X-100 in PBS for $8 \mathrm{~min}$. Primary and secondary antibodies were diluted in PBS containing $0.2 \%$ BSA. Antibody dilutions were as follows: 1:200 monoclonal anti- $\alpha$-actinin (sarcomeric, clone EA-53 from Sigma-Aldrich) and 1:300 anti-myomesin (mMaC, Developmental Studies Hybridoma Bank, University of Iowa). Specific secondary antibodies conjugated to Alexa Fluor 647 (Life Technologies) were used at a dilution of 1:250. After incubation with primary antibodies at room temperature for $30 \mathrm{~min}$, dishes were washed at least three times for $10 \mathrm{~min}$ each with PBS containing $0.2 \% \mathrm{BSA}$, and then treated with fluorescent secondary antibodies. After an additional incubation for $30 \mathrm{~min}$ at room temperature, the dishes were washed, and square glass coverslips were mounted using Mowiol containing 10\% DABCO (1,4- diazabicyclo[2.2.2] octane). Plastic dish borders were cut away and the dishes were mounted to slides. Images were acquired with an Orca-Flash4.0 V2 sCMOS camera (Hamamatsu) on an upright Leica DM6000B fluorescence wide-field 
microscope equipped with a HCX PL APO $\times 63 / 1.40-0.60$ oil-immersion objective. Image acquisition was carried out using the LAS X software (Leica). Deconvolution of immunofluorescence images was performed using AutoQuant/AutoDeblur 2D non-blind Deconvolution (AutoQuant Imaging).

Fast Fourier transform analysis. Myofibril line-scans were taken with the program Fiji (ImageJ) for three different channels in cardiomyocytes co-expressing full-length Tmod1, Lmod1 or Lmod2 along with their fragments, and co-stained with anti- $\alpha$-actinin antibodies. One-dimensional fast Fourier transform analysis was performed on individual line-scans with the program Origin (version 7.0). The resulting power spectra from $50(\operatorname{Tmod} 1$ and Lmod2) or $30(\operatorname{Lmod} 1)$ line scans were then averaged with the program Origin.

\section{References}

1. Yamashiro, S., Gokhin, D. S., Kimura, S., Nowak, R. B. \& Fowler, V. M. Tropomodulins: pointed-end capping proteins that regulate actin filament architecture in diverse cell types. Cytoskeleton (Hoboken) 69, 337-370 (2012).

2. Bennett, V. \& Baines, A. J. Spectrin and ankyrin-based pathways: metazoan inventions for integrating cells into tissues. Physiol. Rev. 81, 1353-1392 (2001).

3. Greenfield, N. J., Kostyukova, A. S. \& Hitchcock-DeGregori, S. E. Structure and tropomyosin binding properties of the $\mathrm{N}$-terminal capping domain of tropomodulin 1. Biophys. J. 88, 372-383 (2005).

4. Fowler, V. M., Greenfield, N. J. \& Moyer, J. Tropomodulin contains two actin filament pointed end-capping domains. J. Biol. Chem. 278, 40000-40009 (2003).

5. Kostyukova, A. S., Choy, A. \& Rapp, B. A. Tropomodulin binds two tropomyosins: a novel model for actin filament capping. Biochemistry 45, 12068-12075 (2006)

6. Krieger, I., Kostyukova, A., Yamashita, A., Nitanai, Y. \& Maeda, Y. Crystal structure of the C-terminal half of tropomodulin and structural basis of actin filament pointed-end capping. Biophys. J. 83, 2716-2725 (2002).

7. Rao, J. N., Madasu, Y. \& Dominguez, R. Mechanism of actin filament pointed-end capping by tropomodulin. Science 345, 463-467 (2014).

8. Conley, C. A., Fritz-Six, K. L., Almenar-Queralt, A. \& Fowler, V. M. Leiomodins: larger members of the tropomodulin (Tmod) gene family. Genomics 73, 127-139 (2001).

9. Nanda, V. \& Miano, J. M. Leiomodin 1, a new serum response factordependent target gene expressed preferentially in differentiated smooth muscle cells. J. Biol. Chem. 287, 2459-2467 (2012).

10. Yuen, M. et al. Leiomodin-3 dysfunction results in thin filament disorganization and nemaline myopathy. J. Clin. Invest. 124, 4693-4708 (2014).

11. Chereau, D. et al. Leiomodin is an actin filament nucleator in muscle cells. Science 320, 239-243 (2008).

12. Conley, C. A. Leiomodin and tropomodulin in smooth muscle. Am. J. Physiol. Cell Physiol. 280, C1645-C1656 (2001).

13. Chereau, D. et al. Actin-bound structures of Wiskott-Aldrich syndrome protein (WASP)-homology domain 2 and the implications for filament assembly. Proc. Natl Acad. Sci. USA 102, 16644-16649 (2005).

14. Dominguez, R. The beta-thymosin/WH2 fold: multifunctionality and structure. Ann. NY Acad. Sci. 1112, 86-94 (2007).

15. Dominguez, R. Structural insights into de novo actin polymerization. Curr. Opin. Struct. Biol. 20, 217-225 (2010).

16. Cenik, B. K. et al. Severe myopathy in mice lacking the MEF2/SRF-dependent gene leiomodin-3. J. Clin. Invest. 125, 1569-1578 (2015).

17. Weber, A., Pennise, C. R., Babcock, G. G. \& Fowler, V. M. Tropomodulin caps the pointed ends of actin filaments. J. Cell Biol. 127, 1627-1635 (1994).

18. Pollard, T. D. \& Cooper, J. A. Quantitative analysis of the effect of Acanthamoeba profilin on actin filament nucleation and elongation. Biochemistry 23, 6631-6641 (1984).

19. Skwarek-Maruszewska, A. et al. Different localizations and cellular behaviors of leiomodin and tropomodulin in mature cardiomyocyte sarcomeres. Mol. Biol. Cell 21, 3352-3361 (2010).
20. von der Ecken, J. et al. Structure of the F-actin-tropomyosin complex. Nature 519, 114-117 (2015).

21. Bella, J., Hindle, K. L., McEwan, P. A. \& Lovell, S. C. The leucine-rich repeat structure. Cell. Mol. Life. Sci. 65, 2307-2333 (2008).

22. Tsukada, T. et al. Identification of residues within tropomodulin-1 responsible for its localization at the pointed ends of the actin filaments in cardiac myocytes. J. Biol. Chem. 286, 2194-2204 (2011).

23. Boczkowska, M., Rebowski, G., Kast, D. J. \& Dominguez, R. Structural analysis of the transitional state of Arp2/3 complex activation by two actin-bound WCAs. Nat. Commun. 5, 3308 (2014).

24. Pardee, J. D. \& Spudich, J. A. Purification of muscle actin. Methods Enzymol. 85 Pt B, 164-181 (1982).

25. Smillie, L. B. Preparation and identification of alpha- and beta-tropomyosins Methods Enzymol. 85 Pt B, 234-241 (1982).

26. Adams, P. D. et al. PHENIX: a comprehensive Python-based system for macromolecular structure solution. Acta Crystallogr. D Biol. Crystallogr. 66 , 213-221 (2010).

27. Emsley, P., Lohkamp, B., Scott, W. G. \& Cowtan, K. Features and development of Coot. Acta Crystallogr. D Biol. Crystallogr. 66, 486-501 (2010).

28. Skwarek-Maruszewska, A., Hotulainen, P., Mattila, P. K. \& Lappalainen, P. Contractility-dependent actin dynamics in cardiomyocyte sarcomeres. J. Cell Sci. 122, 2119-2126 (2009).

29. Valdar, W. S. Scoring residue conservation. Proteins 48, 227-241 (2002).

\section{Acknowledgements}

This work was supported by National Institutes of Health grant R01 GM073791 to R.D. and a grant from the Finnish Foundation for Cardiovascular Research to P.L. We thank Benjamin L. Prosser for helpful discussions about the analysis of the cellular data.

\section{Author contributions}

All the authors participated in the design of the experiments and the conception of the project; M.B. and G.R. produced all the proteins and constructs for cell expression, conducted actin polymerization assays, ITC experiments, crystallization and X-ray data collection, and participated in the analysis of the cellular data; E.K. and P.L. performed and analysed cellular experiments; R.D. refined the crystal structures; M.B., G.R. and R.D. prepared the figures, and R.D. wrote the paper.

\section{Additional information}

Accession codes: The atomic coordinates and structure factors for the structures of Lmod1 $1_{\mathrm{ABS} 2}, \mathrm{TL} 1_{\mathrm{ABS} 2}$ and GS1-TL1 $1_{\mathrm{ABS} 2}$ :actin have been deposited in the Protein Data Bank with accession codes $4 \mathrm{Z79}, 4 \mathrm{Z8G}$ and $4 \mathrm{Z94}$, respectively.

Supplementary Information accompanies this paper at http://www.nature.com/ naturecommunications

Competing financial interests: The authors declare no competing financial interests.

Reprints and permission information is available online at http://npg.nature.com/ reprintsandpermissions/

How to cite this article: Boczkowska, M. et al. How Leiomodin and Tropomodulin use a common fold for different actin assembly functions. Nat. Commun. 6:8314 doi: $10.1038 /$ ncomms9314 (2015)

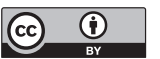

This work is licensed under a Creative Commons Attribution 4.0 International License. The images or other third party material in this article are included in the article's Creative Commons license, unless indicated otherwise in the credit line; if the material is not included under the Creative Commons license, users will need to obtain permission from the license holder to reproduce the material. To view a copy of this license, visit http://creativecommons.org/licenses/by/4.0/ 